\title{
Asset Allocation and Liquidity Breakdowns: What if Your Broker Does not Answer the Phone?
}

ABSTRACT: This paper analyzes the portfolio decision of an investor facing the threat of illiquidity. In a continuous-time setting, the efficiency loss due to illiquidity is addressed and quantified. We show that the efficiency loss for a logarithmic investor with 30 years until the investment horizon is a significant $22.7 \%$ of current wealth if the illiquidity part of the model is calibrated to the Japanese data of the aftermath of WW II. Furthermore, it is demonstrated that the threat of illiquidity can change the demand for risky securities tremendously and that a logarithmic investor will not behave myopically anymore.

Keywords: Illiquidity, Blackout Period, Portfolio Decision, Efficiency Loss, Rare Disasters

JEL-Classification: G11 


\section{Introduction}

Over decades the assumption that investors can trade continuously has been central to the theory of modern finance. In the history of trading at the stock exchanges, there are however examples where liquidity tried out or trading has been (virtually) not possible for a number of reasons, including political turmoil, war, or hyperinflation. For instance, after World War II, the Tokyo stock exchange was closed from August 1945 until May 1949 and it reopened with a loss of $95 \%$ compared to the pre-war stock prices. Besides, the stock exchanges of European countries that had been invaded by Germany were closed down for some months. The same is true for the German stock exchanges that were closed for at least sixth months. Even the Swiss Stock Exchange closed from 10 May 1940 until 8 June 1940 and reopened with a loss of over 20\%. Similarly, during the First World War several European stock exchanges were temporarily closed and even the NYSE closed for 4.5 months (31 July 1914 - 28 November 1914). Recently, the NYSE was closed for four days after the terrorist attacks of 9/11 and reopened on 17 September setting a record volume of 2.37 billion shares. The US stock market lost almost $10 \%$ of its value. This example shows that trading breaks can induce strong wishes to rebalance portfolios and may be accompanied by sharp price drops. The goal of this paper is to analyze the portfolio decision of an investor facing the threat of trading interruptions. As documented in the data, we allow for jumps in the market prices at the advent and the end of a trading interruption. The following table summarizes some examples. ${ }^{1}$

\begin{tabular}{c|c|c} 
Exchange & Trading Break & Comment \\
\hline London & $07 / 1914-01 / 1915$ & WW I \\
New York & $08 / 1914-11 / 1914$ & WW I \\
Zurich & $05 / 1940-06 / 1940$ & WW II (Mobilization) \\
Frankfurt & $04 / 1945-09 / 1945$ & Aftermath of WW II \\
Tokyo & $08 / 1945-05 / 1949$ & Aftermath of WW II \\
New York & $09 / 11 / 2001-09 / 14 / 2001$ & Terrorist Attack
\end{tabular}

Table 1: Examples for Major Trading Breaks

There are several related papers modeling liquidity effects explicitly. One strand of literature weakens the assumption that investors are price takers. In these models, trading takes place continuously, but large traders cannot trade without inducing price impacts. Papers dealing

\footnotetext{
${ }^{1}$ See, e.g., Frey and Kucher (1999), Jorion and Goetzman (1999), Siegel (2002). The are several other examples. For instance, Jorion and Goetzman report that in Germany, Italy, and German-occupied territories dealing in shares was subject to strict controls during WW II leading to a sharp fall in liquidity.
} 
with this issue include Bank and Baum (2002) and Cetin et al. (2004), among others. A second strand of literature introduces transaction costs into the model implying that it is not optimal for investors to trade continuously. Papers in this area include Duffie and Sun (1990), Davis and Norman (1990), and Korn (1998), among others. Besides, Longstaff (2001) looks at the portfolio problem of an investor who can only implement portfolio strategies with finite variation and thus faces liquidity constraints. Schwartz and Tebaldi (2006) assume that an investor cannot trade a risky asset at all, i.e. the trading interruption is permanent. Examples are human wealth or housing. Closely related to our paper are the papers by Kahl et al. (2003) and Longstaff (2005). Kahl et al. (2003) consider an investor's portfolio problem where the advent of a trading interruption is known and Longstaff (2005) analyzes the implications for the equilibrium prices of assets in such a setting. The problem presented in Kahl et al. (2003) can be solved recursively (firstly solve the problem in the absorbing state and then solve the problem for the non-absorbing state by using the solution of the absorbing state as boundary condition). In contrast to that, to the best of our knowledge, our paper is the first one solving a problem where both states (trading and non-trading state) are recurrent. This is particularly relevant from an economical point of view, since during political turmoils or wars markets may close and reopen several times and nobody knows in advance how often this will take place. For instance, after $9 / 11$ it was not obvious whether a second wave of terrorist attacks could soon hit the United States of America. Besides, the problem is mathematically more involved, since it cannot be solved recursively any more.

Furthermore, our paper is also related to the asset pricing literature dealing with the equity premium puzzle. In particular, Rietz (1988) and recently Barro (2005) point out that the puzzle can (partly) be resolved if investors take into consideration the potential for a rare economic disaster occurring with a small probability. Barro (2005) writes that "a worthwhile extension would deal more seriously with the dynamics of crisis regimes". Since we model two distinct regimes explicitly (normal and illiquidity), our paper also contributes to this strand of literature. Additionally, a later section demonstrates how our model can be extended to include three regimes (normal, crisis, illiquidity).

Our paper contributes to the existing literature in multiple ways: The efficiency loss due to illiquidity is addressed and we are able to quantify the impact of illiquidity on an investor's portfolio decision. We show that the efficiency loss for a logarithmic investor with 30 years until the investment horizon is a significant $22.7 \%$ of current wealth if the illiquidity part of the model is calibrated to the Japanese data of the aftermath of WW II. Besides, we demonstrate that the threat of illiquidity can change the demand for risky securities tremendously. Finally, we are able to solve a continuous-time multi-state portfolio problem and present an (almost) closed-form solution to a system of coupled Hamilton-Jacobi-Bellman equations.

The remainder of the paper is structured as follows. Section 2 describes the continuous-time framework. Section 3 introduces the investor's portfolio problem and derives its solution. In 
Section 4, our results are illustrated by numerical examples and the efficiency loss due to illiquidity is analyzed. Section 5 briefly discusses an extension of our model to a setting with three regimes. Section 6 concludes. All proofs can be found in the Appendix.

\section{Continuous-Time Framework}

We consider a simple two-asset securities market. The first asset is a (locally risk-free) money market account and the second one is risky (stock or stock index). The economic regimes are characterized by a finite set of states, where, by convention, 0 is the initial state at time 0 . The process $\mathcal{Z}(t)$ denotes the state at time $t$ and we assume that $\mathcal{Z}$ is a right-continuous process with left limits. The associated $(J+1)$-dimensional counting process $N=\left(N^{k}\right)_{k \in \mathcal{J}}$ defined by

$$
N^{k}(t)=\#\{s \mid s \in(0, t], \mathcal{Z}(s-) \neq k, \mathcal{Z}(s)=k\}
$$

is also right-continuous with left limits. Note that $N^{k}$ counts the number of transitions into state $k$. For reasons that become clear later on, we do not restrict our model to only two states. ${ }^{2}$ The money market account (syn. bond) and the stock index (syn. stock) possess the dynamics

$$
d M=M r_{\mathcal{Z}} d t
$$

$M(0)=1$, and $^{3}$

$$
d S=S^{-}\left[\mu_{\mathcal{Z}} d t+\sigma_{\mathcal{Z}} d W-\sum_{k \neq \mathcal{Z}^{-}} L_{\mathcal{Z}^{-} k} d N^{k}\right],
$$

$S(0)>0$, where $W$ is a Brownian motion and we allow the interest rate $r_{\mathcal{Z}}$, the expected mean $\mu_{\mathcal{Z}}$, and the volatility $\sigma_{\mathcal{Z}}$ to depend on the economic regime. The constant $L_{j k}$ models the jump size of the stock price upon transition from state $j$ into state $k$. The wealth dynamics of an investor putting his funds into bonds and stocks read

$$
d X=X^{-}\left[\left(r_{\mathcal{Z}}+\pi \alpha_{\mathcal{Z}}\right) d t+\pi \sigma_{\mathcal{Z}} d W-\sum_{k \neq \mathcal{Z}^{-}} \pi L_{\mathcal{Z}^{-} k} d N^{k}\right]
$$

where $\alpha_{\mathcal{Z}}=\mu_{\mathcal{Z}}-r_{\mathcal{Z}}$ denotes the excess return and $\pi$ denotes the proportion of wealth invested in stocks. Denoting the number of shares of the risky asset by $\varphi$, we thus have $\pi=\varphi S / X$. We restrict our set of admissible trading strategies to those satisfying $\pi \in[0,1]$, i.e. short sales of the risky asset are prohibited. ${ }^{4}$ This assumption also excludes any strategy that allows the possibility of zero wealth, which is in line with Dybvig and Huang (1988). Furthermore, it is

\footnotetext{
${ }^{2}$ For instance, jumps within a state can be modeled by an enlargement of the state space such that a two state model turns into a four state model. This is so because a Poisson process can be represented by a two-state Markov chain.

${ }^{3}$ To shorten notation, we usually write $Y$ instead of $Y(t)$ and $Y^{-}$instead $Y(t-)$ if $Y$ is a stochastic process.

${ }^{4}$ Proposition 3.2 provides a sufficient condition such that short sales are not optimal. Our numerical results show that for reasonable parametrizations of the model short sales are not optimal.
} 
assumed that the investor maximizes expected utility from terminal wealth at final time $T$ with respect to a logarithmic utility function $U(x)=\ln (x)$. Such an investor is characterized by the fact that, in general, he makes myopic portfolio decisions if he can trade continuously. As will be shown later on, this result breaks down if he faces the threat of illiquidity.

As in Merton $(1969,1971)$, in normal times trading takes place continuously. However, there is a threat that a catastrophic event like a war or a terrorist attack hits the economy triggering a shutdown of the exchange where the risky asset is traded. In this case, the investor is not able to buy or sell his assets and is forced to stick to the portfolio that he has chosen before the liquidity breakdown. If the investor reaches his retirement age while the exchange is closed, then he can liquidate his risky assets only by suffering a loss of $l \varphi S=l \pi X$, where $l \in[0,1]$ denotes the loss rate. For instance, if $l=1$, then the investor would lose all his money invested in stocks. We emphasize that, as long as the exchange is closed, the proportion invested in stocks is not a choice variable, but exogenously given. The following lemma provides an explicit solution for its dynamics.

Lemma 2.1 ( $\pi$-dynamics) For a fixed number of stocks, the proportion invested in stocks follows the dynamics

$$
d \pi=\pi^{-}\left(1-\pi^{-}\right)\left[\left(\alpha_{\mathcal{Z}}-\sigma_{\mathcal{Z}}^{2} \pi\right) d t+\sigma_{\mathcal{Z}} d W-\sum_{k \neq \mathcal{Z}^{-}} \frac{L_{\mathcal{Z}^{-k}}}{1-\pi^{-} L_{\mathcal{Z}^{-k}}} d N^{k}\right] .
$$

This stochastic differential equation (SDE) has the closed-form solution $\pi(t)=1 /(1+Z(t))$ with

$$
d Z=Z^{-}\left[\left(\sigma_{\mathcal{Z}}^{2}-\alpha_{\mathcal{Z}}\right) d t-\sigma_{\mathcal{Z}} d W+\sum_{k \neq \mathcal{Z}^{-}} \frac{L_{\mathcal{Z}^{-k}}}{1-L_{\mathcal{Z}^{-k}}} d N^{k}\right] .
$$

Remark. This lemma shows that $\pi$ remains between 0 and 1 if this is so for the initial value and the loss rates $L_{j k}$. Note that $Z$ becomes a geometric Brownian motion if the loss rates are zero.

\section{Portfolio Decision}

By assumption, the investor maximizes expected utility from terminal wealth with respect to a logarithmic utility function $U(x)=\ln (x)$. We firstly consider a model with two regimes, normal (state 0 ) and illiquidity (state 1), and assume that trading comes to a halt in the illiquidity regime. ${ }^{5}$ The liquidity breakdown occurs with intensity $\lambda_{01}$, whereas a subsequent recovery of the economy can happen with intensity $\lambda_{10}$. We allow the stock dynamics to follow a jumpdiffusion process in state 0 and denote the corresponding jump size by $L_{0}$ and the jump intensity

\footnotetext{
${ }^{5}$ In a later section, we include a crisis regime in which trading is still possible. This could be done by adding a state to the state space $\mathcal{J}$.
} 
by $\lambda_{0} \cdot{ }^{6}$ Upon transition into the illiquidity state, the stock price may also jump by $L_{01}$ and the diffusion parameters change from $\alpha_{0}$ and $\sigma_{0}$ into $\alpha_{1}$ and $\sigma_{1}$. In the illiquidity state, the stock also follows a jump-diffusion and the corresponding jump is denoted by $L_{1}$ and the jump intensity by $\lambda_{1}$.

[INSERT FIGURE 1 ABOUT HERE]

Denoting the investor's indirect utility function (syn. value function) in the normal regime $\mathrm{by}^{7}$

$$
J^{0}(t, x)=\max _{\pi} \mathrm{E}_{0}^{t, x}\left[U\left(X^{\pi}(T)\right)\right]
$$

it thus satisfies the Hamilton-Jacobi-Bellman equation (HJB)

$$
\begin{aligned}
0= & \max _{\pi}\left\{J_{t}^{0}+x\left(r_{0}+\alpha_{0} \pi\right) J_{x}^{0}+0.5 x^{2} \pi^{2}\left(\sigma_{0}\right)^{2} J_{x x}^{0}\right. \\
& \left.+\lambda_{01}\left[J^{1}\left(t, x\left(1-\pi L_{01}\right), \frac{\left(1-L_{01}\right) \pi}{1-L_{01} \pi}\right)-J^{0}(t, x)\right]+\lambda_{0}\left[J^{0}\left(t, x\left(1-\pi L_{0}\right)\right)-J^{0}(t, x)\right]\right\}
\end{aligned}
$$

with terminal condition $J^{0}(T, x)=U(x)$. Here,

$$
J^{1}(t, x, \pi)=\mathrm{E}_{1}^{t, x, \pi}\left[U\left(X^{\pi}(T)\right)\right]
$$

denotes the investor's indirect utility function in the illiquidity regime (state 1). We emphasize that, in this regime, the proportion invested in stock, $\pi$, is not at the discretionary of the investor any more and thus becomes a state variable. ${ }^{8}$ This also implies that the last argument of $J^{1}$ in the HJB (2) equals $\frac{\left(1-L_{01}\right) \pi}{1-L_{01} \pi}$, since

$$
\pi=\frac{\varphi S}{X}=\frac{\varphi S^{-}\left(1-L_{01}\right)}{X^{-}\left(1-\pi^{-} L_{01}\right)}=\pi^{-} \frac{\left(1-L_{01}\right)}{1-L_{01} \pi^{-}}
$$

The HJB in the illiquidity state reads

$$
\begin{aligned}
0= & J_{t}^{1}+x\left(r_{1}+\alpha_{1} \pi\right) J_{x}^{1}+0.5 x^{2} \pi^{2}\left(\sigma_{1}\right)^{2} J_{x x}^{1}+x \pi^{2}(1-\pi)\left(\sigma_{1}\right)^{2} J_{x \pi}^{1} \\
& +\pi(1-\pi)\left(\alpha_{1}-\left(\sigma_{1}\right)^{2} \pi\right) J_{\pi}^{1}+0.5 \pi^{2}(1-\pi)^{2}\left(\sigma_{1}\right)^{2} J_{\pi \pi}^{1} \\
& +\lambda_{10}\left[J^{0}\left(t, x\left(1-\pi L_{10}\right)\right)-J^{1}(t, x, \pi)\right] \\
& +\lambda_{1}\left[J^{1}\left(t, x\left(1-\pi L_{1}\right), \frac{\pi\left(1-L_{1}\right)}{1-\pi L_{1}}\right)-J^{1}(t, x, \pi)\right]
\end{aligned}
$$

with terminal condition $J^{1}(T, x, \pi)=U(x(1-l \pi))$, where $l$ models liquidation costs. We conjecture $J^{0}(t, x)=\ln (x)+f^{0}(t)$ and $J^{1}(t, x, \pi)=\ln (x)+f^{1}(t, \pi)$ with $f^{0}(T)=0$ and

\footnotetext{
${ }^{6}$ From now one, we use a mixture of a jump-diffusion model and a two-state Markov chain for notational convenience. As mentioned in Section 2, the jumps can equivalently be modeled by enlarging the state space of the Markov chain, i.e. by considering a four state Markov chain.

${ }^{7}$ The notation $\mathrm{E}_{j}^{t, x}$ means that expectation is taken under the assumption that at time $t$ the Markov chain is in state $j$ and that the investor's current wealth equals $x$.

${ }^{8}$ Note the difference between the state variables of an HJB and the state of a Markov chain.
} 
$f^{1}(T, \pi)=\ln (1-l \pi)$. Then HJB $(2)$ becomes

$$
\begin{aligned}
0= & \max _{\pi}\left\{f_{t}^{0}+r_{0}+\alpha_{0} \pi-0.5 \pi^{2}\left(\sigma_{0}\right)^{2}\right. \\
& \left.+\lambda_{01}\left[\ln \left(1-\pi L_{01}\right)+f^{1}\left(t, \frac{\left(1-L_{01}\right) \pi}{1-L_{01} \pi}\right)-f^{0}(t)\right]+\lambda_{0} \ln \left(1-\pi L_{0}\right)\right\} .
\end{aligned}
$$

This leads to the following first-order condition (FOC) for the optimal stock proportion $\pi^{*}$ in state 0 .

$$
0=\alpha_{0}-\sigma_{0}^{2} \pi^{*}-\lambda_{01} \frac{L_{01}}{1-\pi^{*} L_{01}}+\lambda_{01} f_{\pi}^{1}\left(t, \frac{\left(1-L_{01}\right) \pi^{*}}{1-L_{01} \pi^{*}}\right) \frac{1-L_{01}}{\left(1-L_{01} \pi^{*}\right)^{2}}-\lambda_{0} \frac{L_{0}}{1-\pi^{*} L_{0}} .
$$

It is well-known that, in general, a logarithmic investor makes his investment decisions myopically if continuous-trading is possible. The FOC, however, shows that the threat of illiquidity turns this myopic behavior into a non-myopic one. Note that $\pi^{*}$ is a deterministic function of time $t$. We shall later prove that under some technical conditions the FOC has a unique solution. Furthermore, (3) becomes

$$
\begin{aligned}
0= & f_{t}^{1}-\lambda_{10} f^{1}+\pi(1-\pi)\left(\alpha_{1}-\left(\sigma_{1}\right)^{2} \pi\right) f_{\pi}^{1}+0.5 \pi^{2}(1-\pi)^{2}\left(\sigma_{1}\right)^{2} f_{\pi \pi}^{1} \\
& +\lambda_{1}\left[f^{1}\left(t, \frac{\pi\left(1-L_{1}\right)}{1-\pi L_{1}}\right)-f^{1}(t, \pi)\right]+g_{1}(\pi)+\lambda_{10} f^{0},
\end{aligned}
$$

where $g_{1}(y)=r_{1}+\alpha_{1} y-0.5 y^{2}\left(\sigma_{1}\right)^{2}+\lambda_{1} \ln \left(1-y L_{1}\right)+\lambda_{10} \ln \left(1-y L_{10}\right)$ is a real-valued function. For this reason, we get the following stochastic representations for $f^{1}$ and $f_{\pi}^{1}$.

Proposition 3.1 (Indirect Utility in the Illiquidity Regime) (i) The function $f^{1}$ possesses the stochastic representation

$$
f^{1}(t, \pi)=\int_{t}^{T}\left(\lambda_{10} f^{0}(s)+\mathrm{E}_{1}^{t, \pi}\left[g_{1}(\tilde{\pi}(s))\right]\right) e^{-\lambda_{10}(s-t)} d s+\mathrm{E}_{1}^{t, \pi}[\ln (1-l \tilde{\pi}(T))] e^{-\lambda_{10}(T-t)},
$$

where $\tilde{\pi}(s)=\pi /(\pi+(1-\pi) \tilde{Z}(s))$,

$$
d \tilde{Z}=\tilde{Z}^{-}\left[\left(\sigma_{1}^{2}-\alpha_{1}\right) d s-\sigma_{1} d W+\frac{L_{1}}{1-L_{1}} d \hat{N}\right], \quad \tilde{Z}(t)=1,
$$

and $\hat{N}$ is a Poisson process with intensity $\lambda_{1}$.

(ii) The derivative $f_{\pi}^{1}$ is given by

$$
\frac{\partial f^{1}(t, \pi)}{\partial \pi}=\int_{t}^{T} \mathrm{E}_{1}^{t, \pi}\left[\frac{\partial \tilde{\pi}(s)}{\partial \pi} g_{1}^{\prime}(\tilde{\pi}(s))\right] e^{-\lambda_{10}(s-t)} d s-\mathrm{E}_{1}^{t, \pi}\left[\frac{\partial \tilde{\pi}(T)}{\partial \pi} \frac{l}{1-l \tilde{\pi}(T)}\right] e^{-\lambda_{10}(T-t)},
$$

where $\partial \tilde{\pi}(s) / \partial \pi=\tilde{Z}(s) /(\pi+(1-\pi) \tilde{Z}(s))^{2}$ denotes the derivative of the process $\tilde{\pi}$ with respect to its initial value $\tilde{\pi}(t)=\pi$. Consequently, the derivative $f_{\pi}^{1}$ does not depend on $f^{0}$ and thus (5) provides an algebraic equation for $\pi^{*}$.

Remarks. a) Note that $\tilde{\pi}$ is an auxiliary process with dynamics

$$
d \tilde{\pi}=\tilde{\pi}(1-\tilde{\pi})\left[\left(\alpha_{1}-\sigma_{1}^{2} \tilde{\pi}\right) d t+\sigma_{1} d W-\frac{L_{1}}{1-\tilde{\pi} L_{1}} d \hat{N}\right], \quad \tilde{\pi}(t)=\pi(t)=\pi,
$$


and that $\tilde{Z}(s)=e^{\left(0.5 \sigma_{1}^{2}-\alpha_{1}\right)(s-t)-\sigma_{1}(W(s)-W(t))} /\left(1-L_{1}\right)^{(\hat{N}(s)-\hat{N}(t))}$.

b) In order to calculate $f_{\pi}^{1}$ numerically, the following representation is useful.

$$
\begin{aligned}
\frac{\partial f^{1}(t, \pi)}{\partial \pi}= & \sum_{n=0}^{\infty} \int_{t}^{T} e^{-\lambda_{10}(s-t)} p_{n}(t, s) \int_{-\infty}^{+\infty} \frac{\partial \tilde{\pi}_{n}(t, s, \omega)}{\partial \pi} g_{1}^{\prime}\left(\tilde{\pi}_{n}(t, s, \omega)\right) \psi_{s-t}(\omega) d \omega d s \\
& -e^{-\lambda_{10}(T-t)} \sum_{n=0}^{\infty} p_{n}(t, T) \int_{-\infty}^{+\infty} \frac{\partial \tilde{\pi}_{n}(t, T, \omega)}{\partial \pi} \frac{l}{1-l \tilde{\pi}_{n}(t, T, \omega)} \psi_{T-t}(\omega) d \omega
\end{aligned}
$$

where $^{9}$

$$
\begin{aligned}
g_{1}^{\prime}(y) & =\alpha_{1}-\left(\sigma_{1}\right)^{2} y-\lambda_{1} \frac{L_{1}}{1-y L_{1}}-\lambda_{10} \frac{L_{10}}{1-y L_{10}}, \quad y \in[0,1] \\
p_{n}(t, s) & =P(\hat{N}(s-t)=n)=\frac{e^{-\lambda_{1}(s-t)}\left(\lambda_{1}(s-t)\right)^{n}}{n !}, \\
\psi_{u}(\omega) & =\frac{1}{\sqrt{2 \pi u}} e^{-\frac{\omega^{2}}{2 u}} \\
\tilde{\pi}_{n}(t, s, \omega) & =\frac{\pi}{\pi+(1-\pi) \tilde{z}_{n}(t, s, \omega)}, \\
\frac{\partial \tilde{\pi}_{n}(t, s, \omega)}{\partial \pi} & =\frac{\tilde{z}_{n}(t, s, \omega)}{\left(\pi+(1-\pi) \tilde{z}_{n}(t, s, \omega)\right)^{2}}, \\
\tilde{z}_{n}(t, s, \omega) & =\frac{e^{\left(0.5 \sigma_{1}^{2}-\alpha_{1}\right)(s-t)-\sigma_{1} \omega}}{\left(1-L_{1}\right)^{n}} .
\end{aligned}
$$

One central motivation for modeling the randomness of stock dynamics via Brownian motions is that continuous trading activity of market participants creates this kind of dynamics. ${ }^{10}$ In state 1, however, trading is interrupted and thus it seems to be reasonable to set the diffusion term in state 1 to zero. Besides, we think of state 1 as a regime where the economy is hit by an extreme event such as a war or a political turmoil. Consequently, it might also be plausible to assume that $\alpha_{1} \leq 0$. As the following proposition shows, these assumptions together with (6) are sufficient for the existence of a unique smooth solution of the investor's portfolio choice problem.

Proposition 3.2 (Optimal Portfolio) Assume that $\alpha_{1} \leq 0$ and $\sigma_{1}=0$.

(i) The investor's indirect utility in state 1 is decreasing and concave, i.e. the derivatives $f_{\pi}^{1}$ and $f_{\pi \pi}^{1}$ are negative.

(ii) If there exists ${ }^{11}$ a solution $\pi^{*}$ to the FOC (5), then $\pi^{*}$ is unique and corresponds to the investor's optimal portfolio strategy. Besides, $\pi^{*}$ is a deterministic function of time that is continuously differentiable.

\footnotetext{
${ }^{9}$ In the definition of $\psi_{u}$, the variable $\pi$ equals the constant $3.141 \ldots$ and should not be mixed up with the stock proportion.

${ }^{10}$ See, e.g., Foellmer and Schweizer (1994) and the references therein.

${ }^{11}$ Formally, this means that for each $t \in[0, T]$ there exists a $\pi^{*}(t) \in[0,1]$ such that $\pi^{*}(t)$ solves the FOC (5).
} 
(iii) A solution exists if the right-hand side of the FOC is positive for $\pi=0$ and negative for $\pi=1$, i.e. for all $t \in[0, T]$

$$
\begin{aligned}
\alpha_{0}-\lambda_{0} L_{0}-\lambda_{01} L_{01}+\lambda_{01} f_{\pi}^{1}(t, 0)\left(1-L_{01}\right) & \geq 0 \\
\alpha_{0}-\sigma_{0}^{2}-\lambda_{0} \frac{L_{0}}{1-L_{0}}-\lambda_{01} \frac{L_{01}}{1-L_{01}}+\lambda_{01} f_{\pi}^{1}(t, 1) \frac{1}{1-L_{01}} & \leq 0 .
\end{aligned}
$$

Remark. Condition (6) can be rewritten more explicitly as

$$
\begin{aligned}
0 \leq & \alpha_{0}-\lambda_{0} L_{0}-\lambda_{01} L_{01}-\lambda_{01}\left(1-L_{01}\right) l \mathrm{E}_{1}^{t, 0}[1 / \tilde{Z}(T)] e^{-\lambda_{10}(T-t)} \\
& +\lambda_{01}\left(1-L_{01}\right)\left(\alpha_{1}-\lambda_{1} L_{1}-\lambda_{10} L_{10}\right) \int_{t}^{T} \mathrm{E}_{1}^{t, 0}[1 / \tilde{Z}(s)] e^{-\lambda_{10}(s-t)} d s \\
0 \geq & \alpha_{0}-\sigma_{0}^{2}-\lambda_{0} \frac{L_{0}}{1-L_{0}}-\lambda_{01} \frac{L_{01}}{1-L_{01}}-\lambda_{01} \frac{1}{1-L_{01}} \frac{l}{1-l} \mathrm{E}_{1}^{t, 1}[\tilde{Z}(T)] e^{-\lambda_{10}(T-t)} \\
& +\lambda_{01} \frac{1}{1-L_{01}}\left(\alpha_{1}-\sigma_{1}^{2}-\lambda_{1} \frac{L_{1}}{1-L_{1}}-\lambda_{10} \frac{L_{10}}{1-L_{10}}\right) \int_{t}^{T} \mathrm{E}_{1}^{t, 1}[\tilde{Z}(s)] e^{-\lambda_{10}(s-t)} d s .
\end{aligned}
$$

Note that the assumptions $\alpha_{1} \leq 0$ and $\sigma_{1}=0$ are by no means necessary, but they imply that $f_{\pi}^{1}$ and $f_{\pi \pi}^{1}$ are negative, which are the key properties used in our proof. Besides, we remark that condition (6) is satisfied for reasonable choices of $\alpha_{0}$. However, if $\alpha_{0}$ is "too large" or "too small", then it can happen that this condition is not satisfied. For instance, if $\alpha_{0}$ is negative, i.e. an investment in stocks is strictly inferior compared to an investment in bonds, then the optimal proportion held in stocks is zero, which is a corner solution. These kinds of degenerated cases are excluded by (6). The following proposition provides an explicit representation of the investor's indirect utility function in the normal state.

Proposition 3.3 (Indirect Utility in the Normal Regime) The investor's indirect utility in state 0 is given by

$$
J^{0}(t, x)=\ln (x)+\frac{\lambda_{01}}{\lambda_{01}+\lambda_{10}} e^{\left(\lambda_{01}+\lambda_{10}\right) t} \int_{t}^{T} F(s) e^{-\lambda_{01} s} d s+\frac{\lambda_{10}}{\lambda_{01}+\lambda_{10}} \int_{t}^{T} F(s) e^{\lambda_{10} s} d s,
$$

where

$$
\begin{gathered}
F(t)=g_{0}\left(\pi^{*}(t)\right) e^{-\lambda_{10} t}+\lambda_{01} \int_{t}^{T} \mathrm{E}_{1}^{t, \hat{\pi}_{0}(t)}\left[g_{1}(\tilde{\pi}(s))\right] e^{-\lambda_{10} s} d s \\
+\lambda_{01} \mathrm{E}_{1}^{t, \hat{\pi}_{0}(t)}[\ln (1-l \tilde{\pi}(T))] e^{-\lambda_{10} T}
\end{gathered}
$$

and $\hat{\pi}_{0}(t)=\left(1-L_{01}\right) \pi^{*}(t) /\left(1-L_{01} \pi^{*}(t)\right), g_{0}\left(\pi^{*}\right)=r_{0}+\alpha_{0} \pi^{*}-0.5\left(\pi^{*}\right)^{2}\left(\sigma_{0}\right)^{2}+\lambda_{0} \ln \left(1-\pi^{*} L_{0}\right)+$ $\lambda_{01} \ln \left(1-\pi^{*} L_{01}\right)$.

Usually, one has to solve the integrals in the representation for $J^{0}$ numerically. There is however a particular situation where an explicit formula for the investor's indirect utility is achieved. This is the case for $\sigma_{1}=\alpha_{1}=L_{1}=L_{0}=L_{01}=0$. Then the FOC simplifies into

$$
0=\alpha_{0}-\sigma_{0}^{2} \pi^{*}+\lambda_{01}\left(\left(e^{-\lambda_{10}(T-t)}-1\right) \frac{L_{10}}{1-\pi^{*} L_{10}}-e^{-\lambda_{10}(T-t)} \frac{l}{1-\pi^{*} l}\right) .
$$


If additionally $l=L_{10}$, then we get

$$
0=\alpha_{0}-\sigma_{0}^{2} \pi^{*}-\lambda_{01} \frac{L_{10}}{1-\pi^{*} L_{10}}
$$

and the investor's indirect utility is explicitly given by

$$
\begin{aligned}
f^{0}(t)= & \frac{\lambda_{01}}{\lambda_{01}+\lambda_{10}} e^{\left(\lambda_{01}+\lambda_{10}\right) t}\left(\left(g_{0}\left(\pi^{*}\right)+\frac{\lambda_{01}}{\lambda_{10}} g_{1}\left(\pi^{*}\right)\right) \frac{1}{\lambda_{01}+\lambda_{10}}\left(e^{-\left(\lambda_{01}+\lambda_{10}\right) t}-e^{-\left(\lambda_{01}+\lambda_{10}\right) T}\right)\right. \\
& \left.+\left(\ln \left(1-L_{10} \pi^{*}\right)-\frac{1}{\lambda_{10}} g_{1}\left(\pi^{*}\right)\right) e^{-\lambda_{10} T}\left(e^{-\lambda_{01} t}-e^{-\lambda_{01} T}\right)\right) \\
& +\frac{\lambda_{10}}{\lambda_{01}+\lambda_{10}}\left(\left(\lambda_{01} \ln \left(1-L_{10} \pi^{*}\right)-\frac{\lambda_{01}}{\lambda_{10}} g_{1}\left(\pi^{*}\right)\right) e^{-\lambda_{10} T} \frac{1}{\lambda_{10}}\left(e^{\lambda_{10} T}-e^{\lambda_{10} t}\right)\right. \\
& \left.+\left(g_{0}\left(\pi^{*}\right)+\frac{\lambda_{01}}{\lambda_{10}} g_{1}\left(\pi^{*}\right)\right)(T-t)\right),
\end{aligned}
$$

We now shift our attention to the important question of whether the Hamilton-Jacobi-Bellman equations have smooth solutions. This is established in the following proposition.

Proposition 3.4 (Classical Solutions) Under the assumptions of Proposition 3.2, the functions $f^{0}$ and $f^{1}$ are classical solutions of the HJBs, i.e. $f^{0} \in C^{1}$ and $f^{1} \in C^{1,2}$.

In our applications, we wish to quantify the utility loss of an investor facing the threat of illiquidity. For the reader's convenience, we briefly establish the investor's indirect utility function in the case that trading is allowed in both states. The investor's indirect utility functions in states $j \in\{0,1\}$ are then given by $J^{j}(t, x)=\max _{\pi} \mathrm{E}_{j}^{t, x}\left[U\left(X^{\pi}(T)\right)\right]$ leading to the following result.

Proposition 3.5 (Optimal Solution without Illiquidity) The first-order condition for the investor's optimal portfolio strategy $\pi_{j}^{*}$ in state $j \in\{0,1\}$ is given by

$$
0=\alpha_{j}-\pi_{j}^{*} \sigma_{j}^{2}-\lambda_{j} \frac{L_{j}}{1-\pi_{j}^{*} L_{j}}-\lambda_{j k} \frac{L_{j k}}{1-\pi_{j}^{*} L_{j k}} .
$$

The investor's indirect utility in state 0 reads

$$
J^{0}(t, x)=\ln (x)+\frac{\lambda_{01}}{\lambda_{01}+\lambda_{10}} e^{\left(\lambda_{10}+\lambda_{01}\right) t} \int_{t}^{T} F(s) e^{-\lambda_{01} s} d s+\frac{\lambda_{10}}{\lambda_{01}+\lambda_{10}} \int_{t}^{T} F(s) e^{\lambda_{10} s} d s
$$

where

$$
\begin{aligned}
F(t) & =g_{0}\left(\pi_{0}^{*}\right) e^{-\lambda_{10} t}+\frac{\lambda_{01}}{\lambda_{10}} g_{1}\left(\pi_{1}^{*}\right)\left(e^{-\lambda_{10} t}-e^{-\lambda_{10} T}\right), \\
g_{j}(y) & =r_{j}+\alpha_{j} y-0.5 y^{2} \sigma_{j}^{2}+\lambda_{j} \ln \left(1-y L_{j}\right)+\lambda_{j k} \ln \left(1-y L_{j k}\right) .
\end{aligned}
$$




\section{Numerical Illustrations}

One important example for a major trading break is the aftermath of World War II in Japan. At that time, the Tokyo Stock Exchange was shut down for almost four years reopening with a loss of more than 90\%. Rietz (1988) and Barro (2005), among others, emphasize that these kinds of events can have a significant impact on security prices in an economy. For this reason, we wish to quantify the investor's utility gain expressed in terms of his initial capital when he is able to trade even in the illiquidity state. More precisely, we calculate the amount of initial capital that he would be willing to pay in order to be able to trade in all states. Table 2 summarizes our numerical results for different parametrizations of the model. The column " $\Delta x(\%)$ " contains the percentage by which the initial capital of 100 can be reduced in order to get the same utility as in the model where trading is allowed in both states (see Proposition 3.5).

[INSERT TABLE 2 ABOUT HERE]

We assume that the stock dynamics follow a diffusion process in state 0 and are deterministic in state 1 , since $\sigma_{0}=0.25, L_{0}=0$ and $\sigma_{1}=0, L_{1}=0 .{ }^{12}$ However, either when entering or when leaving state 1 the stock loses a fraction of its value, i.e. either $L_{01}>0$ or $L_{10}>0$. The parameters $\lambda_{01}$ and $\lambda_{10}$ are chosen in order to mimic situations such as in Japan after WW II. For instance, the parametrization $\lambda_{01}=0.01, \lambda_{10}=0.3, L_{01}=0$, and $L_{10}=0.9$ implies that, on average, once in a century the illiquidity state is reached and, on average, this state is left after 3.33 years triggering a stock price decrease of $90 \%$. In this particular case, for an an investment horizon of $T=30$ years, the change of initial capital amounts to over $22 \%$. This is due to the fact that an investor who is able to trade can avoid the loss that is triggered by a jump from state 1 to state 0 . He will sell his stocks once the economy is in state 1 and thus use the money market account as a "safe heaven". If the investor cannot trade, he will not be able to avoid this loss. For this reason, he invests considerably less of his wealth into the risky asset. Figure 2 depicts the function $f_{\pi}^{1}(0, \cdot)$, as well as the right-hand side of the FOC (5) as a function of $\pi$ with $t=0$. The column labeled by $\pi_{0, i l l i q}^{*}$ contains the optimal time-0 stock demands in state 0 (liquidity) when the investment horizon is $T$ and trading is not possible once the economy has jumped into state 1 . In contrast to these demands, if trading is allowed in state 1 , then the investor behaves myopically and the optimal stock demand is $\pi_{0, l i q}^{*}=80 \%$ except for the cases where $L_{10}=0$. As mentioned above, we have $\pi_{1, l i q}^{*}=0$.

[INSERT FIGURE 2 ABOUT HERE]

As $\lambda_{01}$ is much smaller than $\lambda_{10}$, at time $T$, we expect the economy to be in state 0 . Therefore, setting $l=0$ has only a small impact on the percental change of initial capital, which can be

\footnotetext{
${ }^{12}$ Drift and interest rate are in line with Barro (2005).
} 
seen in the third and fourth line of Table 2. However, if the loss rate $L_{10}$ increases from $50 \%$ to $90 \%$, then the percental change of initial capital increases significantly. Increasing $\lambda_{10}$ to 1 only results in small change indicating that the effect of illiquidity is small if the investor does not suffer additional losses. The percental change of the initial capital, however, strongly depends on the intensity $\lambda_{01}$ modeling the probability that the exchange is closed. If we interchange the values of $L_{01}$ and $L_{10}$ as well as $\lambda_{01}$ and $\lambda_{10}$, then the change of initial capital is zero. In this case, the investor will not hold any risky assets in state 0 independent of whether he can trade or not. This is so because short-sales are not allowed. Therefore, an investor who is exposed to the threat of illiquidity will not have any disadvantage. If we only interchange the values of $L_{01}$ and $L_{10}$, we get much smaller percental changes of the initial capital. In this situation, both investors will hold less stocks in state 0 . It is also interesting to note that in this case, the percental change of initial capital decreases when the loss rate increases. For a higher loss rate $L_{10}$, an investor being able to trade loses more money when the economy switches to state 1 . Thus he will benefit less from being able to trade in the illiquidity state 1 . Note that, in the case of $\alpha_{1}=0$, we are in the situation of the example described in Section 3 and thus have an explicit formula for the investor's indirect utility.

\section{[INSERT FIGURE 3 ABOUT HERE]}

For our second application, we consider a model where the indirect utilities of states 0 and 1 are identical if the investor can trade in both states. This allows us to quantify the mere effect of illiquidity. As depicted in Figure 3, we thus construct a version of our two-state model in the following way: Consider an ordinary model where the stock dynamic follow a pure diffusion process (Merton model). The corresponding variables are denoted by $r_{M}, \sigma_{M}, \alpha_{M}$. Obviously, such a model yields the same indirect utility as our two-state model if the investor can trade in both states, all jumps are set to zero, and $r_{0}=\hat{r}_{1}=r_{M}, \sigma_{0}=\hat{\sigma}_{1}=\sigma_{M}, \alpha_{0}=\hat{\alpha}_{1}=\alpha_{M}$. We now wish to construct a pure jump model for state 1 that leads to the same indirect utility. We thus set the volatility in state 1 equal to zero and allow for stock price jumps in state 1 . The variables of the pure jump model for state 1 are denoted by $r_{1}, \sigma_{1}=0, \alpha_{1}, \lambda_{1}$ and $L_{1}$. Using the conjecture for the indirect utility functions, we now compare the following HJBs for the diffusion and the pure jump model.

$$
\begin{aligned}
0 & =\max _{\hat{\pi}_{1}}\left\{\hat{f}_{t}^{1}+\hat{r}_{1}+\hat{\pi}_{1} \hat{\alpha}_{1}-0.5 \hat{\pi}_{1}^{2} \hat{\sigma}_{1}^{2}\right\} \\
0 & =\max _{\pi_{1}}\left\{f_{t}^{1}+r_{1}+\pi_{1} \alpha_{1}+\lambda_{1} \ln \left(1-\pi_{1} L_{1}\right)\right\} .
\end{aligned}
$$

Note that the HJBs do not contain a jump term since, by assumption, $f^{0}=\hat{f}^{1}=f^{1}$ and the loss rate upon transition from state 1 to state 0 equals zero. Differentiating with respect to $\hat{\pi}_{1}$ and $\pi_{1}$, respectively, yields

$$
\hat{\pi}_{1}^{*}=\frac{\hat{\alpha}_{1}}{\hat{\sigma}_{1}^{2}}, \quad \pi_{1}^{*}=\frac{1}{L_{1}}-\frac{\lambda_{1}}{\alpha_{1}} .
$$


Substituting these optimal strategies into the equations (7), the indirect utility functions in the two states are identical if the parameters $\alpha_{1}, \lambda_{1}$, and $L_{1}$ satisfy the following requirement.

$$
\hat{r}_{1}+\frac{\hat{\alpha}_{1}^{2}}{2 \hat{\sigma}_{1}^{2}}=r_{1}+\frac{\alpha_{1}}{L_{1}}+\lambda_{1}\left(\ln \left(\frac{\lambda_{1} L_{1}}{\alpha_{1}}\right)-1\right) .
$$

For simplicity, we set $\alpha_{1}=\hat{\alpha}_{1}, r_{1}=\hat{r}_{1}$. Then for fixed $L_{1}$, one can solve the above equation for $\lambda_{1}$. For different parametrization of the model, Table 3 provides the changes of the initial capital. It can be seen that now the effect of illiquidity is smaller. It increases with $\lambda_{01}$ and decreases with $\lambda_{10}$. As Table 4 shows, the impact of illiquidity becomes much more relevant if the loss rate $l$ is not equal to zero, i.e. if the investor loses a fraction of his funds invested in stocks given that his investment horizon is reached while the exchange is closed. However, for $l \neq 0$, the model is no longer equivalent to the original Merton model. As before, throughout Table 3 and Table 4 we have $\pi_{0, l i q}^{*}=80 \%$.

[INSERT TABLES 3 and 4 ABOUT HERE]

\section{Three Regimes}

As a generalization of the model presented in Section 3, we now consider an economy with three regimes, normal (state 0), illiquidity (state 1 ) and a third regime (state 2) in which excess return and volatility can be different from the corresponding parameters in state 0 . For instance, state 2 can model an economic crisis where trading is possible, but the excess return is lower and the volatility is higher as in the normal state. According to Barro (2005), this is a relevant extension of the model.

In each state, the stock follows a jump-diffusion process where $\alpha_{i}$ denotes the excess return and $\sigma_{i}$ denotes the volatility, $i \in\{0,1,2\}$. The size of a relative stock price jump in state $i$ is denoted by $L_{i}$ and its intensity by $\lambda_{i}$. Besides, $\lambda_{i j}$ stands for the intensity for a regime change from state $i$ into state $j$. The corresponding loss rate is denoted by $L_{i j}$. As before, the investor is not allowed to buy or sell his assets, while the economy is in the illiquidity regime (state 1).

\section{[INSERT FIGURE 4 ABOUT HERE]}

The investor's indirect utility functions are now given by

$$
J^{j}(t, x)=\max _{\pi} \mathrm{E}_{j}^{t, x}\left[U\left(X^{\pi}(T)\right)\right], j \in\{0,2\}, \quad J^{1}(t, x, \pi)=\mathrm{E}_{1}^{t, x, \pi}\left[U\left(X^{\pi}(T)\right)\right] .
$$

For $j, k \in\{0,2\}$ with $j \neq k$, we obtain the following Hamilton-Jacobi-Bellman equations for the three states.

$0=\max _{\pi_{j}}\left\{J_{t}^{j}(t, x)+x\left(r_{j}+\pi_{j} \alpha_{j}\right) J_{x}^{j}(t, x)+0.5 x^{2} \pi_{j}^{2} \sigma_{j}^{2} J_{x x}^{j}(t, x)\right.$ 


$$
\begin{aligned}
& +\lambda_{j}\left[J^{j}\left(t, x\left(1-\pi_{j} L_{j}\right)\right)-J^{j}(t, x)\right] \\
& \left.+\lambda_{j 1}\left[J^{1}\left(t, x\left(1-\pi_{j} L_{j 1}\right), \frac{\left(1-L_{j 1}\right) \pi_{j}}{1-L_{j 1} \pi_{j}}\right)-J^{j}(t, x)\right]+\lambda_{j k}\left[J^{k}\left(t, x\left(1-\pi_{j} L_{j k}\right)\right)-J^{j}(t, x)\right]\right\}, \\
0= & J_{t}^{1}(t, x, \pi)+x\left(r_{1}+\pi \alpha_{1}\right) J_{x}^{1}(t, x, \pi)+0.5 x^{2} \pi^{2} \sigma_{1}^{2} J_{x x}^{1}(t, x, \pi)+x \pi^{2}(1-\pi) \sigma_{1}^{2} J_{x, \pi}^{1}(t, x, \pi) \\
& +\pi(1-\pi)\left(\alpha_{1}-\sigma_{1}^{2} \pi\right) J_{\pi}^{1}(t, x, \pi)+0.5 \pi^{2}(1-\pi)^{2} \sigma_{1}^{2} J_{\pi \pi}^{1}(t, x, \pi) \\
& +\lambda_{1}\left[J^{1}\left(t, x\left(1-\pi L_{1}\right), \frac{\left(1-L_{1}\right) \pi}{1-L_{1} \pi}\right)-J^{1}(t, x, \pi)\right] \\
& +\lambda_{10}\left[J^{0}\left(t, x\left(1-\pi L_{10}\right)\right)-J^{1}(t, x, \pi)\right]+\lambda_{12}\left[J^{2}\left(t, x\left(1-\pi L_{12}\right)\right)-J^{1}(t, x, \pi)\right],
\end{aligned}
$$

with terminal conditions $J^{j}(T, x)=U(x)$ and $J^{1}(t, x)=U(x(1-l \pi))$, where $l$ again models liquidation costs. Setting

$$
g_{j}(y)=r_{j}+\alpha_{j} y-0.5 y^{2} \sigma_{j}^{2}+\lambda_{j} \ln \left(1-y L_{j}\right)+\sum_{j \neq k \in\{0,1,2\}} \lambda_{j k} \ln \left(1-y L_{j k}\right), \quad j \in\{0,1,2\},
$$

we conjecture

$$
J^{0}(t, x)=\ln (x)+f^{0}(t), \quad J^{1}(t, x, \pi)=\ln (x)+f^{1}(t, \pi), \quad J^{2}(t, x)=\ln (x)+f^{2}(t)
$$

implying

$$
\begin{aligned}
0= & \max _{\pi_{j}}\left\{f_{t}^{j}(t)+g_{j}\left(\pi_{j}\right)+\lambda_{j 1}\left[f^{1}\left(t, \frac{\left(1-L_{j 1}\right) \pi_{j}}{1-L_{j 1} \pi_{j}}\right)-f^{j}(t)\right]+\lambda_{j k}\left[f^{k}(t)-f^{j}(t)\right]\right\}, \\
0= & f_{t}^{1}(t, \pi)+\pi(1-\pi)\left(\alpha_{1}-\sigma_{1}^{2} \pi\right) f_{\pi}^{1}(t, \pi)+0.5 \pi^{2}(1-\pi)^{2} \sigma_{1}^{2} f_{\pi \pi}^{1}(t, \pi)+g_{1}(\pi) \\
& +\lambda_{1}\left[f^{1}\left(t, \frac{\pi\left(1-L_{1}\right)}{1-\pi L_{1}}\right)-f^{1}(t, \pi)\right]+\lambda_{10}\left[f^{0}(t)-f^{1}(t, \pi)\right]+\lambda_{12}\left[f^{2}(t)-f^{1}(t, \pi)\right],
\end{aligned}
$$

for $j, k \in\{0,2\}$ with $j \neq k$. This leads to the following first order conditions for the investor's optimal portfolio strategies in states 0 and $2, \pi_{0}^{*}$ and $\pi_{2}^{*}$.

$$
0=\alpha_{j}-\sigma_{j}^{2} \pi_{j}^{*}-\lambda_{j} \frac{L_{j}}{1-\pi_{j}^{*} L_{j}}-\lambda_{j 1} \frac{L_{j 1}}{1-\pi_{j}^{*} L_{j 1}}-\lambda_{j k} \frac{L_{j k}}{1-\pi_{j}^{*} L_{j k}}+\lambda_{j 1} f_{\pi}^{1}\left(t, \frac{\left(1-L_{j 1}\right) \pi_{j}^{*}}{1-L_{j 1} \pi_{j}^{*}}\right) \frac{1-L_{j 1}}{\left(1-L_{j 1} \pi_{j}^{*}\right)^{2}}
$$

As before, $\pi_{0}^{*}$ and $\pi_{2}^{*}$ are deterministic functions of time $t$ that only depend on $f_{\pi}^{1}$. Furthermore, we get the following stochastic representation for $f^{1}$ and $f_{\pi}^{1}$.

Proposition 5.1 (Indirect Utility in the Illiquidity Regime) (i) The function $f^{1}$ reads

$$
\begin{aligned}
f^{1}(t, \pi)= & \int_{t}^{T}\left(\lambda_{10} f^{0}(s)+\lambda_{12} f^{2}(s)+\mathrm{E}_{1}^{t, \pi}\left[g_{1}(\tilde{\pi}(s))\right]\right) e^{-\left(\lambda_{10}+\lambda_{12}\right)(s-t)} d s \\
& +\mathrm{E}_{1}^{t, \pi}[\ln (1-l \tilde{\pi}(T))] e^{-\left(\lambda_{10}+\lambda_{12}\right)(T-t)},
\end{aligned}
$$

where $\tilde{\pi}(s)=\pi /(\pi+(1-\pi) \tilde{Z}(s))$,

$$
d \tilde{Z}=\tilde{Z}^{-}\left[\left(\sigma_{1}^{2}-\alpha_{1}\right) d s-\sigma_{1} d W+\frac{L_{1}}{1-L_{1}} d N_{1}\right], \quad \tilde{Z}(t)=1
$$

and $N_{1}$ is a Poisson process with intensity $\lambda_{1}$.

(ii) The derivative $f_{\pi}^{1}$ is given by

$$
\frac{\partial f^{1}(t, \pi)}{\partial \pi}=\int_{t}^{T} \mathrm{E}_{1}^{t, \pi}\left[\frac{\partial \tilde{\pi}(s)}{\partial \pi} g_{1}^{\prime}(\tilde{\pi}(s))\right] e^{-\left(\lambda_{10}+\lambda_{12}\right)(s-t)} d s-\mathrm{E}_{1}^{t, \pi}\left[\frac{\partial \tilde{\pi}(T)}{\partial \pi} \frac{l}{1-l \tilde{\pi}(T)}\right] e^{-\left(\lambda_{10}+\lambda_{12}\right)(T-t)},
$$


where $\partial \tilde{\pi}(s) / \partial \pi=\tilde{Z}(s) /(\pi+(1-\pi) \tilde{Z}(s))^{2}$ denotes the derivative of $\tilde{\pi}(s)$ with respect to its initial value $\tilde{\pi}(t)=\pi$.

This time, substituting $f_{\pi}^{1}$ into the HJB equations for states 0 and 2 yields a linear system of two second-order differential equations. As opposed to the setting of only two different states, by reduction to first-order, we now end up with a four-dimensional system of first-order ODEs. We are able to explicitly determine the roots of the corresponding characteristic polynomial of order four and thus may derive a representation of $f^{1}$ by applying the variation of constants method and Cramer's rule. The following proposition provides such an explicit representation of the investor's indirect utility function in the normal regime if $\lambda_{01}=\lambda_{21}$. As mentioned before, we think of state 1 as being triggered by a catastrophic event leading to a closure of the stock, whereas state 2 corresponds to an economic crisis during which the investor can still trade. Thus, $\lambda_{01}=\lambda_{21}$ means that the occurrence of a catastrophic event does not depend on whether the economy is currently in crisis or not.

Proposition 5.2 (Indirect Utility in Normal Regime) Assume that $\lambda_{01}=\lambda_{21}$ and let $\lambda_{j} .=\sum_{j \neq k} \lambda_{j k}$ denote the aggregate intensity of leaving state $j$. The investor's indirect utility in state 0 is given by

$$
\begin{aligned}
J^{0}(t, x)= & \ln (x)+e^{\lambda_{1} \cdot t}\left(c_{1} \int_{t}^{T} e^{\left(\lambda_{02}+\lambda_{2} \cdot-\lambda_{1} \cdot\right)(t-s)}\left(F_{0}(s)-F_{2}(s)\right) d s\right. \\
& +c_{2} \int_{t}^{T} e^{\lambda_{21}(t-s)}\left(\left(\lambda_{10}-\lambda_{20}\right) F_{0}(s)+\left(\lambda_{12}-\lambda_{02}\right) F_{2}(s)\right) d s \\
& \left.+c_{3} \int_{t}^{T} e^{-\lambda_{1 \cdot}(t-s)}\left(\left(\lambda_{12} \lambda_{20}+\lambda_{10} \lambda_{2} .\right) F_{0}(s)+\left(\lambda_{02} \lambda_{1}+\lambda_{12} \lambda_{21}\right) F_{2}(s)\right) d s\right),
\end{aligned}
$$

where

$$
F_{j}(t)=g_{i}\left(\pi_{j}^{*}(t)\right) e^{-\lambda_{1} \cdot t}+\lambda_{j 1} \int_{t}^{T} \mathrm{E}_{1}^{t, \hat{\pi}_{j}(t)}\left[g_{1}(\tilde{\pi}(s))\right] e^{-\lambda_{1} \cdot s} d s+\lambda_{j 1} \mathrm{E}_{1}^{t, \hat{\pi}_{j}(t)}[\ln (1-l \tilde{\pi}(T))] e^{-\lambda_{1} \cdot T}
$$

and $\hat{\pi}_{j}(t)=\left(1-L_{j 1}\right) \pi_{j}^{*}(t) /\left(1-L_{j 1} \pi_{j}^{*}(t)\right)$. The constants $c_{j}$ are given by (15) (see Appendix).

Remark. In the proof of the previous proposition, we also provide a representation for $J^{0}$ if $\lambda_{01} \neq \lambda_{21}$.

The function $J^{2}$ possesses a similar representation as $J^{0}$. Therefore, as in the setting with two states, we get that $f^{0}$ and $f^{2}$ are continuously differentiable. Furthermore, by the Feynman-Kac representation of $f^{1}$, we obtain that $f^{1} \in C^{1,2}$. Hence the functions $f^{0}, f^{1}$, and $f^{2}$ are classical solutions of the corresponding HJBs. 


\section{Conclusion}

This paper studies the portfolio decision of an investor facing the threat of illiquidity. Illiquidity is understood as a state in which the investor is not able to trade at all. Calibrating the illiquidity part of the dynamics of the risky asset to the Japanese data of the aftermath of WW II, it is shown that this threat has a significant effect on the investor's portfolio decision and that the efficiency loss is remarkable $22.7 \%$ of current wealth if the investment horizon is 30 years and the investor has logarithmic utility. To obtain these results, we solve the corresponding control problem explicitly, which means that we derive the solution to a system of coupled HamiltonJacobi-Bellman equations. Our paper also contributes to the literature dealing with the equity premium puzzle, since we introduce a model that is able to address the time dimension of an economic crisis in which trading is not possible. We note, however, that our model is of partial equilibrium type and thus our numerical results should be viewed as suggestive rather than

definitive. One possible direction for future research might be to study a general equilibrium model with multiple agents. 


\section{References}

Bank, P. and D. Baum (2004): Hedging and Portfolio Optimization in Financial Markets with a Large Trader, Mathematical Finance 14, 118.

Barro, R. J. (2005): Rare disasters and asset markets in the twentieth century, Working Paper, Havard University.

Cetin, U.; R. Jarrow and P. Protter (2004): Liquidity risk and arbitrage pricing theory, Finance and Stochastics 8, 311341.

Davis, M. H. A. and A. R. Norman (1990): Portfolio selection with transaction costs, Mathematics of Operations Research 15, 676-713.

Duffie, D. and T.-S. Sun (1990): Transactions Costs and Portfolio Choice in a Discrete-Continuous Time Setting, Journal of Economic Dynamics and Control 14, 35-51.

Frey, B. S. and M. Kucher (1999): History as reflected in capital markets: The Case of World War II, Working Paper, University of Zurich.

Jorion, P. and W. N. Goetzmann (1999): Global stock markets in the twentieth century, Journal of Finance 54, 953-980.

Kahl, M.; J. Liu and F. A. Longstaff (2003): Paper millionaires: how valuable is stock to a stockholder who is restricted from selling it, Journal of Financial Economics 67, 385-410.

Korn, R. (1998): Portfolio Optimisation with Strictly Positive Transaction Costs and Impulse Control, Finance and Stochastics 2, 85-114.

Longstaff, F. A. (2001): Optimal Portfolio Choice and the Valuation of Illiquid Securities, Review of Financial Studies 14, 407-431.

Longstaff, F. A. (2005): Asset pricing in markets with illiquid assets, Working Paper, UCLA.

Merton, R. C. (1969): Lifetime portfolio selection under uncertainty: the continuous case, Reviews of Economical Statistics 51, 247-257.

Merton, R. C. (1971): Optimal consumption and portfolio rules in a continuous-time model, Journal of Economic Theory 3, 373-413.

Rietz, T. A. (1988): The equity premium: A solution, Journal of Monetary Economics 22, 117-131.

Schwartz, E. S. and C. Tebaldi (2006): Illiquid Assets and Optimal Portfolio Choice, Working Paper, UCLA and NBER.

Siegel, J. J. (2002): Stocks for the long run, McGraw-Hill, New York. 


\section{A Appendix}

Proof of Lemma 2.1. Set $Y=1 / X$. We have

$$
d Y=Y^{-}\left[\left(-r_{\mathcal{Z}}-\pi \alpha_{\mathcal{Z}}+\pi^{2} \sigma_{\mathcal{Z}}^{2}\right) d t-\pi \sigma_{\mathcal{Z}} d W+\sum_{k \neq \mathcal{Z}^{-}} \frac{\pi^{-} L_{\mathcal{Z}^{-}}}{1-\pi^{-} L_{\mathcal{Z}-k}} d N^{k}\right] .
$$

Note that $\Delta S \Delta Y$ is given by

$$
\Delta S \Delta Y=-S^{-} Y^{-} \sum_{k \neq \mathcal{Z}^{-}} \frac{\pi^{-}\left(L_{\mathcal{Z}^{-}}\right)^{2}}{1-\pi^{-} L_{\mathcal{Z}^{-}}} d N^{k} .
$$

Thus, by the product rule $d(S Y)=S^{-} d Y+Y^{-} d S+d\langle S, Y\rangle^{c}+\Delta S \Delta Y$, we obtain

$$
d(S Y)=S^{-} Y^{-}\left[\left(\alpha_{\mathcal{Z}}(1-\pi)-\sigma_{\mathcal{Z}}^{2} \pi(1-\pi)\right) d t+\sigma_{\mathcal{Z}}(1-\pi) d W-\sum_{k \neq \mathcal{Z}^{-}} \frac{\left(1-\pi^{-}\right) L_{\mathcal{Z}^{-k}}}{1-\pi^{-} L_{\mathcal{Z}}-k} d N^{k}\right]
$$

and the first result follows from $d \pi=\varphi d(S Y)$. The second result follows by an application of Itô's lemma to $Z=1 / \pi-1$.

Proof of Proposition 3.1. The first part follows from Fubini's theorem applied to the Feynman-Kac representation of $f^{1}$ with $f^{1}(T, \pi)=\ln (1-l \pi)$. As for the second part, note that for $0 \leq L_{10}, L_{1}<1$ we have

$$
\left|g_{1}^{\prime}(y)\right| \leq\left|\alpha_{1}\right|+\sigma_{1}^{2}+\lambda_{1} \frac{L_{1}}{1-L_{1}}+\lambda_{10} \frac{L_{10}}{1-L_{10}}
$$

for all $y \in[0,1]$. Acting on the tacit assumption that $\pi \in[0,1]$, by the remark following Lemma 2.1 we obtain

$$
\left|\frac{\partial \tilde{\pi}(\pi, s, \omega)}{\partial \pi} g_{1}^{\prime}(\tilde{\pi}(\pi, s, \omega))\right| \leq \frac{\tilde{Z}(s, \omega)}{(\tilde{Z}(s, \omega) \wedge 1)^{2}}\left(\left|\alpha_{1}\right|+\sigma_{1}^{2}+\lambda_{1} \frac{L_{1}}{1-L_{1}}+\lambda_{10} \frac{L_{10}}{1-L_{10}}\right)
$$

for all $(s, \omega) \in[t, T] \times \Omega$. Furthermore we have

$$
\left|\frac{\partial \tilde{\pi}(\pi, T, \omega)}{\partial \pi} \frac{l}{1-l \tilde{\pi}(\pi, T, \omega)}\right| \leq \frac{\tilde{Z}(T, \omega)}{(\tilde{Z}(T, \omega) \wedge 1)^{2}} \frac{l}{1-l}
$$

for all $\omega \in \Omega$. Therefore, the discounted left-hand sides of (8) and (9) are uniformly bounded in $\pi$ by integrable functions, and we can thus interchange differentiating and integrating.

Proof of Proposition 3.2. (i) and (ii). Let $\alpha_{1} \leq 0, \sigma_{1}=0, \pi \in[0,1]$ and $0 \leq L_{10}, L_{1}<1$. We have $g_{1}^{\prime}(y) \leq 0$, for all $y \in[0,1]$ and $\partial \tilde{\pi}(s) / \partial \pi \geq 0$, for all $s \in[t, T]$. Thus, by the monotonicity of the integral and the remark after Lemma 2.1 we find $f_{\pi}^{1} \leq 0$. Since $\tilde{Z}(s)=\frac{e^{-\alpha_{1}(s-t)}}{\left(1-L_{1}\right)^{\tilde{N}(s)-\tilde{N}(t)}} \geq 1$, we have

$$
\frac{\partial^{2} \tilde{\pi}(s)}{\partial \pi^{2}}=-2 \frac{\tilde{Z}(s)(1-\tilde{Z}(s))}{(\pi+(1-\pi) \tilde{Z}(s))^{3}} \geq 0 .
$$


Further,

$$
g_{1}^{\prime \prime}(y)=-\lambda_{10} \frac{L_{10}^{2}}{\left(1-y L_{10}\right)^{2}}-\lambda_{1} \frac{L_{1}^{2}}{\left(1-y L_{1}\right)^{2}} \leq 0
$$

and consequently

$$
\begin{gathered}
\frac{\partial}{\partial \pi}\left\{\frac{\partial \tilde{\pi}(s)}{\partial \pi} g_{1}^{\prime}(\tilde{\pi}(s))\right\}=\frac{\partial^{2} \tilde{\pi}(s)}{\partial \pi^{2}} g_{1}^{\prime}(\tilde{\pi}(s))+\left(\frac{\partial \tilde{\pi}(s)}{\partial \pi}\right)^{2} g_{1}^{\prime \prime}(\tilde{\pi}(s)) \leq 0 \\
\frac{\partial}{\partial \pi}\left\{\frac{\partial \tilde{\pi}(T)}{\partial \pi} \frac{l}{1-l \tilde{\pi}(T)}\right\}=\frac{\partial^{2} \tilde{\pi}(T)}{\partial \pi^{2}} \frac{l}{1-l \tilde{\pi}(T)}+\left(\frac{\partial \tilde{\pi}(T)}{\partial \pi}\right)^{2} \frac{l^{2}}{(1-l \tilde{\pi}(T))^{2}} \geq 0 .
\end{gathered}
$$

Since $\tilde{\pi} \in[0,1]$, there exist constants $c_{i} \geq 0,1 \leq i \leq 4$ such that

$$
\left|\frac{\partial}{\partial \pi}\left\{\frac{\partial \tilde{\pi}(\pi, s, \omega)}{\partial \pi} g_{1}^{\prime}(\tilde{\pi}(\pi, s, \omega))\right\}\right| \leq c_{1} \tilde{Z}(s, \omega)(\tilde{Z}(s, \omega)-1)+c_{2} \tilde{Z}(s, \omega)^{2}
$$

for all $(s, \omega) \in[t, T] \times \Omega$ and

$$
\left|\frac{\partial}{\partial \pi}\left\{\frac{\partial \tilde{\pi}(\pi, T, \omega)}{\partial \pi} \frac{l}{1-l \tilde{\pi}(\pi, T, \omega)}\right\}\right| \leq c_{3} \tilde{Z}(T, \omega)(\tilde{Z}(T, \omega)-1)+c_{4} \tilde{Z}(T, \omega)^{2}
$$

for all $\omega \in \Omega$. Thus, the discounted left-hand sides of (10) and (11) are uniformly bounded in $\pi$ by integrable functions, and as in the proof of Proposition 3.1, we can thus interchange differentiating and integrating. This yields

$$
\begin{aligned}
\frac{\partial^{2} f^{1}(t, \pi)}{\partial \pi^{2}}= & \int_{t}^{T} \mathrm{E}_{1}^{t, \pi}\left[\frac{\partial^{2} \tilde{\pi}(s)}{\partial \pi^{2}} g_{1}^{\prime}(\tilde{\pi}(s))+\left(\frac{\partial \tilde{\pi}(s)}{\partial \pi}\right)^{2} g_{1}^{\prime \prime}(\tilde{\pi}(s))\right] e^{-\lambda_{10}(s-t)} d s \\
& -\mathrm{E}_{1}^{t, \pi}\left[\frac{\partial^{2} \tilde{\pi}(T)}{\partial \pi^{2}} \frac{l}{1-l \tilde{\pi}(T)}+\left(\frac{\partial \tilde{\pi}(T)}{\partial \pi}\right)^{2} \frac{l^{2}}{(1-l \tilde{\pi}(T))^{2}}\right] e^{-\lambda_{10}(T-t)} \leq 0 .
\end{aligned}
$$

Taking the derivative with respect to $\pi$ of the right hand side of the FOC we get

$$
\begin{aligned}
0 \geq & -\sigma_{0}^{2}-\lambda_{01} \frac{L_{01}^{2}}{\left(1-\pi^{*} L_{01}\right)^{2}}-\lambda_{0} \frac{L_{0}^{2}}{\left(1-\pi^{*} L_{0}\right)^{2}}+\lambda_{01} f_{\pi \pi}^{1}\left(t, \frac{\left(1-L_{01}\right) \pi^{*}}{1-L_{01} \pi^{*}}\right) \frac{\left(1-L_{01}\right)^{2}}{\left(1-L_{01} \pi^{*}\right)^{4}} \\
& +2 \lambda_{01} f_{\pi}^{1}\left(t, \frac{\left(1-L_{01}\right) \pi^{*}}{1-L_{01} \pi^{*}}\right) \frac{L_{01}\left(1-L_{01}\right)}{\left(1-L_{01} \pi^{*}\right)^{3}} .
\end{aligned}
$$

Thus, the solution of the FOC is unique and satisfies the second-order condition. Furthermore, by the implicit function theorem, we conclude that given the existence of the mapping $\pi^{*}$ : $[0, T] \rightarrow[0,1], t \mapsto \pi^{*}(t)$ where $\pi^{*}(t)$ solves the FOC (5), the mapping $\pi^{*}$ is continuously differentiable and maximizes the HJB (4).

(iii) Under our assumptions, the right hand side of the FOC (5) is continuous and decreasing in $\pi$. Therefore, by the intermediate value theorem, the claim follows.

Proof of Proposition 3.3. Recall, that the HJB equation for state 0 is given by

$$
0=f_{t}^{0}(t)+g_{0}\left(\pi^{*}(t)\right)+\lambda_{01} f^{1}\left(t, \hat{\pi}_{0}(t)\right)-\lambda_{01} f^{0}(t),
$$


where $g_{0}\left(\pi^{*}\right)=r_{0}+\alpha_{0} \pi^{*}-\frac{1}{2} \pi^{* 2} \sigma_{0}^{2}+\lambda_{0} \ln \left(1-\pi^{*} L_{0}\right)+\lambda_{01} \ln \left(1-\pi^{*} L_{01}\right)$. By Proposition 3.1, we have

$$
\begin{gathered}
f^{1}\left(t, \hat{\pi}_{0}(t)\right)=\quad \lambda_{10} \int_{t}^{T} f^{0}(s) e^{-\lambda_{10}(s-t)} d s+\int_{t}^{T} \mathrm{E}_{1}^{t, \hat{\pi}_{0}(t)}\left[g_{1}(\tilde{\pi}(s))\right] e^{-\lambda_{10}(s-t)} d s \\
+\mathrm{E}_{1}^{t, \hat{\pi}_{0}(t)}[\ln (1-l \tilde{\pi}(T))] e^{-\lambda_{10}(T-t)},
\end{gathered}
$$

which yields the following integro-differential equation for $f^{0}$

$$
\begin{aligned}
0= & f_{t}^{0}(t) e^{-\lambda_{10} t}+g_{0}\left(\pi^{*}(t)\right) e^{-\lambda_{10} t}+\lambda_{01} \lambda_{10} \int_{t}^{T} f^{0}(s) e^{-\lambda_{10} s} d s-\lambda_{01} f^{0}(t) e^{-\lambda_{10} t} \\
& +\lambda_{01} \int_{t}^{T} \mathrm{E}_{1}^{t, \hat{\pi}_{0}(t)}\left[g_{1}(\tilde{\pi}(s))\right] e^{-\lambda_{10} s} d s+\lambda_{01} \mathrm{E}_{1}^{t, \hat{\pi}_{0}(t)}[\ln (1-l \tilde{\pi}(T))] e^{-\lambda_{10} T} .
\end{aligned}
$$

Substituting

$$
H(t)=\int_{t}^{T} f^{0}(s) e^{-\lambda_{10} s} d s
$$

into the equation above, we get

$$
\begin{aligned}
0=- & H^{\prime \prime}(t)-\left(\lambda_{10}-\lambda_{01}\right) H^{\prime}(t)+\lambda_{01} \lambda_{10} H(t)+g_{0}\left(\pi^{*}(t)\right) e^{-\lambda_{10} t} \\
& \quad+\lambda_{01} \int_{t}^{T} \mathrm{E}_{1}^{t, \hat{\pi}_{0}(t)}\left[g_{1}(\tilde{\pi}(s))\right] e^{-\lambda_{10} s} d s+\lambda_{01} \mathrm{E}_{1}^{t, \hat{\pi}_{0}(t)}[\ln (1-l \tilde{\pi}(T))] e^{-\lambda_{10} T}
\end{aligned}
$$

Eventually, setting

$$
\begin{gathered}
F(t)=g_{0}\left(\pi^{*}(t)\right) e^{-\lambda_{10} t}+\lambda_{01} \int_{t}^{T} \mathrm{E}_{1}^{t, \hat{\pi}_{0}(t)}\left[g_{1}(\tilde{\pi}(s))\right] e^{-\lambda_{10} s} d s \\
+\lambda_{01} \mathrm{E}_{1}^{t, \hat{\pi}_{0}(t)}[\ln (1-l \tilde{\pi}(T))] e^{-\lambda_{10} T}
\end{gathered}
$$

leads to the following second order linear inhomogenous differential equation

$$
H^{\prime \prime}(t)+\left(\lambda_{10}-\lambda_{01}\right) H^{\prime}(t)-\lambda_{01} \lambda_{10} H(t)=F(t)
$$

with the constraints

$$
H(T)=0, \quad H^{\prime}(T)=0 .
$$

The characteristic equation

$$
\mu^{2}+\left(\lambda_{10}-\lambda_{01}\right) \mu-\lambda_{01} \lambda_{10}=0
$$

has the two roots,

$$
\mu_{1}=\lambda_{01}, \quad \mu_{2}=-\lambda_{10} .
$$

Thus, the exponential ansatz yields the following fundamental system for the homogenous differential equation

$$
u_{1}(t)=e^{\lambda_{01} t}, \quad u_{2}(t)=e^{-\lambda_{10} t}
$$


By means of the method of variation of constants, a particular solution of the differential equation (12) is given by

$$
w(t)=u_{1}(t) \int_{t}^{T} \frac{F(s) u_{2}(s)}{W(s)} d s-u_{2}(t) \int_{t}^{T} \frac{F(s) u_{1}(s)}{W(s)} d s,
$$

where the Wronskian determinant $W$ is given by

$$
W(s)=-\left(\lambda_{01}+\lambda_{10}\right) e^{\left(\lambda_{01}-\lambda_{10}\right) s} .
$$

Note that we have $w(T)=0$ and $w^{\prime}(T)=0$. Thus, the unique solution of the constraint differential equation (12) is given by the particular solution $w$, i.e

$$
H(t)=\int_{t}^{T} \frac{F(s)}{\lambda_{01}+\lambda_{10}} e^{\lambda_{10}(s-t)} d s-\int_{t}^{T} \frac{F(s)}{\lambda_{01}+\lambda_{10}} e^{-\lambda_{01}(s-t)} d s .
$$

Differentiating $H$ we obtain

$$
\begin{aligned}
H^{\prime}(t) & =u_{1}^{\prime}(t) \int_{t}^{T} \frac{F(s) u_{2}(s)}{W(s)} d s-u_{2}^{\prime}(t) \int_{t}^{T} \frac{F(s) u_{1}(s)}{W(s)} d s \\
& =-\lambda_{01} \int_{t}^{T} \frac{F(s)}{\lambda_{01}+\lambda_{10}} e^{-\lambda_{01}(s-t)} d s-\lambda_{10} \int_{t}^{T} \frac{F(s)}{\lambda_{01}+\lambda_{10}} e^{\lambda_{10}(s-t)} d s
\end{aligned}
$$

Further, by definition of $H$ we have

$$
H^{\prime}(t)=-f^{0}(t) e^{-\lambda_{10} t}
$$

and thus, $f^{0}$ is given by

$$
f^{0}(t)=\lambda_{01} e^{\lambda_{10} t} \int_{t}^{T} \frac{F(s)}{\lambda_{01}+\lambda_{10}} e^{-\lambda_{01}(s-t)} d s+\lambda_{10} \int_{t}^{T} \frac{F(s)}{\lambda_{01}+\lambda_{10}} e^{\lambda_{10} s} d s
$$

Proof of Proposition 3.4. By Proposition 3.2 (ii), the function $F$ is continuous. Thus

$$
f^{0}(\cdot)=\frac{\lambda_{01}}{\lambda_{01}+\lambda_{10}} e^{\left(\lambda_{01}+\lambda_{10}\right)} \cdot \int_{\cdot}^{T} F(s) e^{-\lambda_{01} s} d s+\frac{\lambda_{10}}{\lambda_{01}+\lambda_{10}} \int^{T} F(s) e^{\lambda_{10} s} d s
$$

is continuously differentiable. As we have already seen in the proof of Proposition 3.1 and Proposition 3.2 , the function $f^{1}$ is twice continuously differentiable with respect to $\pi$. Further, the Feynman-Kac representation

$$
\begin{aligned}
f^{1}(t, \pi)= & \sum_{n=0}^{\infty} \int_{t}^{T} e^{-\lambda_{10}(s-t)} p_{n}(t, s)\left(\lambda_{10} f^{0}(s)+\int_{-\infty}^{\infty} g_{1}\left(\tilde{\pi}_{n}(t, s, \omega)\right) \psi_{s-t}(\omega) d \omega\right) d s \\
& e^{-\lambda_{10}(T-t)} \sum_{n=0}^{\infty} p_{n}(t, T) \int_{-\infty}^{\infty} \ln \left(1-l \tilde{\pi}_{n}(t, T, \omega)\right) \psi_{T-t}(\omega) d \omega
\end{aligned}
$$

implies that $f^{1}$ is continuously differentiable with respect to $t$. 
Proof of Proposition 3.5. Under the assumption of $J^{j}(t, x)=\ln (x)+f^{j}(t)$, we get the following Hamilton-Jacobi-Bellman equations for $j, k \in\{0,1\}$ with $j \neq k$.

$$
\begin{aligned}
0= & \max _{\pi_{j}}\left\{f_{t}^{j}+r_{j}+\alpha_{j} \pi_{j}-0.5 \pi_{j}^{2} \sigma_{j}^{2}\right. \\
& \left.+\lambda_{j k}\left[\ln \left(1-\pi_{j} L_{j k}\right)+f^{k}-f^{j}\right]+\lambda_{j} \ln \left(1-\pi_{j} L_{j}\right)\right\} .
\end{aligned}
$$

with terminal conditions $J^{j}(T, x)=U(x)$. This leads to the following first order conditions for the investor's optimal portfolio $\pi_{j}^{*}$

$$
0=\alpha_{j}-\pi_{j}^{*} \sigma_{j}^{2}-\lambda_{j} \frac{L_{j}}{1-\pi_{j}^{*} L_{j}}-\lambda_{j k} \frac{L_{j k}}{1-\pi_{j}^{*} L_{j k}} .
$$

Furthermore, we have

$$
f^{j}(t)=\frac{g_{j}\left(\pi_{j}^{*}\right)}{\lambda_{j k}}\left(1-e^{-\lambda_{j k}(T-t)}\right)+\lambda_{j k} \int_{t}^{T} f^{k}(s) e^{-\lambda_{j k}(s-t)} d s
$$

where

$$
g_{j}(y)=r_{j}+\alpha_{j} y-0.5 y^{2} \sigma_{j}^{2}+\lambda_{j} \ln \left(1-y L_{j}\right)+\lambda_{j k} \ln \left(1-y L_{j k}\right) .
$$

Substituting the above representation of $f^{1}$ into the HJB (13) for state 0 , and setting

$$
H(t)=\int_{t}^{T} f^{0}(s) e^{-\lambda_{10} s} d s
$$

we obtain the following second order ordinary differential equation

$$
H^{\prime \prime}(t)+\left(\lambda_{10}-\lambda_{01}\right) H^{\prime}(t)-\lambda_{01} \lambda_{10} H(t)=g_{0}\left(\pi_{0}^{*}\right) e^{-\lambda_{10} t}+\frac{\lambda_{01}}{\lambda_{10}} g_{1}\left(\pi_{1}^{*}\right)\left(e^{-\lambda_{10} t}-e^{-\lambda_{10} T}\right) .
$$

Proof of Proposition 5.1. analogously to the proof of Proposition 3.1.

Proof of Proposition 5.2. By Proposition 5.1 and the HJB equations for $f^{0}$ and $f^{2}$, we obtain the following integro-differential equations

$0=f_{t}^{j}(t) e^{-\lambda_{1} \cdot t}+\lambda_{j 1} \int_{t}^{T}\left(\lambda_{1 j} f^{j}(s)+\lambda_{1 k} f^{k}(s)\right) e^{-\lambda_{1} \cdot s} d s+\lambda_{j k} f^{k}(t) e^{-\lambda_{1} \cdot t}-\lambda_{j} \cdot f^{j}(t) e^{-\lambda_{1} \cdot t}+F_{j}(t)$, $j, k \in\{0,2\}$ with $j \neq k$, where

$$
F_{j}(t)=g_{i}\left(\pi_{j}^{*}(t)\right) e^{-\lambda_{1} \cdot t}+\lambda_{j 1} \int_{t}^{T} \mathrm{E}_{1}^{t, \hat{\pi}_{j}(t)}\left[g_{1}(\tilde{\pi}(s))\right] e^{-\lambda_{1} \cdot s} d s+\lambda_{j 1} \mathrm{E}_{1}^{t, \hat{\pi}_{j}(t)}[\ln (1-l \tilde{\pi}(T))] e^{-\lambda_{1} \cdot T}
$$

and $\hat{\pi}_{j}(t)=\left(1-L_{j 1}\right) \pi_{j}^{*}(t) /\left(1-L_{j 1} \pi_{j}^{*}(t)\right)$. Substituting $H_{i}(t)=\int_{t}^{T} f^{i}(s) e^{-\lambda_{1} \cdot s} d s$ into these equations, we obtain a linear inhomogeneous system of two second order constant-coefficient differential equations

$$
\begin{aligned}
& H_{0}^{\prime \prime}(t)+\left(\lambda_{1}-\lambda_{0 .}\right) H_{0}^{\prime}(t)-\lambda_{01} \lambda_{10} H_{0}(t)-\lambda_{01} \lambda_{12} H_{2}(t)+\lambda_{02} H_{2}^{\prime}(t)=F_{0}(t) \\
& H_{2}^{\prime \prime}(t)+\left(\lambda_{1}-\lambda_{2} .\right) H_{2}^{\prime}(t)-\lambda_{21} \lambda_{12} H_{2}(t)-\lambda_{21} \lambda_{10} H_{0}(t)+\lambda_{20} H_{0}^{\prime}(t)=F_{2}(t)
\end{aligned}
$$


with terminal conditions $H_{0}(T)=0, H_{0}^{\prime}(T)=0, H_{2}(T)=0, H_{2}^{\prime}(T)=0$. By definition of $H_{0}$, the investor's indirect utility in state 0 is given by

$$
J^{0}(t, x)=\ln (x)-H_{0}^{\prime}(t) e^{\lambda_{1} \cdot t}
$$

Setting $\Delta_{j k}=\lambda_{j} .-\lambda_{k}$. and substituting $\left(u_{0}, u_{1}\right)=\left(H_{0}, H_{0}^{\prime}\right)$ and $\left(v_{0}, v_{1}\right)=\left(H_{2}, H_{2}^{\prime}\right)$, the system (14) can be transformed into the following system of first-order ODEs.

$$
\begin{aligned}
u_{0}^{\prime} & =u_{1}, \\
u_{1}^{\prime} & =\lambda_{01} \lambda_{10} u_{0}-\Delta_{10} u_{1}+\lambda_{01} \lambda_{12} v_{0}-\lambda_{02} v_{1}+F_{0}, \\
v_{0}^{\prime} & =v_{1}, \\
v_{1}^{\prime} & =\lambda_{21} \lambda_{10} u_{0}-\lambda_{20} u_{1}+\lambda_{21} \lambda_{12} v_{0}-\Delta_{12} v_{1}+F_{2} .
\end{aligned}
$$

The characteristic polynomial of the corresponding homogenous system reads

$$
p_{h}(\mu)=\mu\left(\mu+\lambda_{1} \cdot\right)\left(\mu^{2}+p \mu+q\right)
$$

where

$$
p=\lambda_{1 .}-\lambda_{0 .}-\lambda_{2 .} \quad q=\lambda_{01}\left(\lambda_{2 .}-\lambda_{10}\right)+\lambda_{21}\left(\lambda_{02}-\lambda_{12}\right) .
$$

Thus, the eigenvalues are explicitly given by $\mu_{1}=0, \mu_{2}=-\lambda_{1}$. and $\mu_{3,4}=-p / 2 \pm \sqrt{\left(p^{2} / 4-q\right)}$. Note that in case of $\lambda_{01}=\lambda_{21}$, we have

$$
\mu_{3}=\lambda_{21} \quad \mu_{4}=\lambda_{0} \cdot-\lambda_{1}+\lambda_{20}
$$

By the variation of constants method and Cramer's rule, the solution of the above system under the constraint $\left(u_{0}, u_{1}, v_{0}, v_{1}\right)^{t}(T)=0$ is given by

$$
(u, v)^{t}(t)=\sum_{j=1}^{4} y_{j}(t) \int_{t}^{T} \frac{-D_{j}(s)}{\operatorname{det}\left(y_{k l}\right)(s)} d s,
$$

where $y_{j}, j=1, \ldots, 4$ is a fundamental system of the corresponding homogenous system and $D_{j}$ denotes the determinant of the matrix $\left(y_{1}, y_{2}, y_{3}, y_{4}\right)$ where the $j$ th column is replaced by $\left(0, F_{0}, 0, F_{2}\right)^{t}$. Note that in case of four pairwise different real eigenvalues, the exponential ansatz yields that a fundamental system is given by $y_{j}=v_{j} e^{\mu_{j}}$, where $v_{j}$ denotes the eigenvector corresponding to $\mu_{j}$. Eventually, for $\lambda_{01}=\lambda_{21}$ we get

$$
\begin{aligned}
H_{0}^{\prime}(t)= & -c_{1} \int_{t}^{T} e^{\left(\lambda_{02}+\lambda_{2} \cdot-\lambda_{1} \cdot\right)(t-s)}\left(F_{0}(s)-F_{2}(s)\right) d s \\
& -c_{2} \int_{t}^{T} e^{\lambda_{21}(t-s)}\left(\left(\lambda_{10}-\lambda_{20}\right) F_{0}(s)+\left(\lambda_{12}-\lambda_{02}\right) F_{2}(s)\right) d s \\
& -c_{3} \int_{t}^{T} e^{-\lambda_{1} \cdot(t-s)}\left(\left(\lambda_{12} \lambda_{20}+\lambda_{10} \lambda_{2}\right) F_{0}(s)+\left(\lambda_{02} \lambda_{1} \cdot+\lambda_{12} \lambda_{21}\right) F_{2}(s)\right) d s,
\end{aligned}
$$


where

$$
\begin{aligned}
c_{1} & =\frac{\left(\lambda_{02}^{2}-\lambda_{12} \lambda_{21}+\lambda_{02}\left(\lambda_{2 .}-\lambda_{1}\right)\right)\left(\lambda_{02} \lambda_{20}-\lambda_{10} \lambda_{2 .}+\lambda_{20}\left(\lambda_{2 .}-\lambda_{12}\right)\right)}{\left(\lambda_{02}-\lambda_{1}+\lambda_{20}\right)\left(\lambda_{02}+\lambda_{2 .}\right)\left(\lambda_{02} \lambda_{20}-\lambda_{10} \lambda_{2 .}+\lambda_{20}\left(\lambda_{2 .}-\lambda_{12}\right)\right)} \\
c_{2} & =\frac{\lambda_{21}}{\left(\lambda_{1 .}-\lambda_{02}-\lambda_{20}\right)\left(\lambda_{1 .}+\lambda_{21}\right)} \\
c_{3} & =\frac{1}{\left(\lambda_{1 .}+\lambda_{21}\right)\left(\lambda_{02}+\lambda_{2 .}\right)}
\end{aligned}
$$




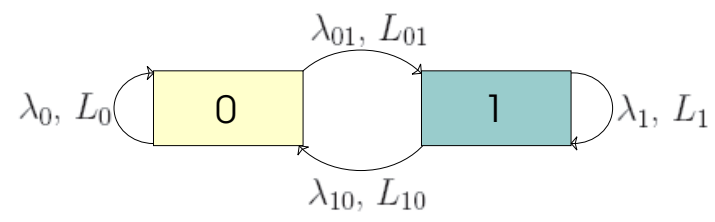

Figure 1: The figure depicts an economy with two regimes, where trading is possible in state 0 and impossible in state 1.

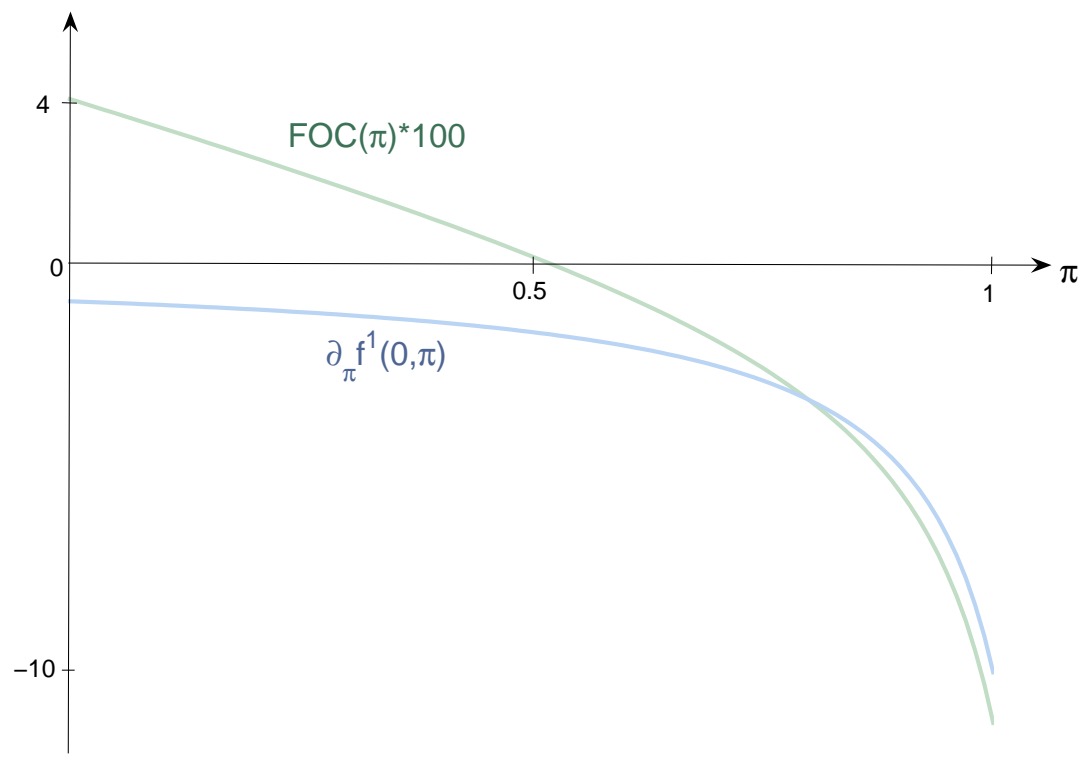

Figure 2: The figure depicts the function $f_{\pi}^{1}(0, \cdot)$, as well as the right-hand side of the FOC (5) as a function of $\pi$, where the parameters are chosen according to the sixth line of table 2 . To get a more pronounced slope, the latter curve is multiplied by 100 . Note that this does not change the null of the function. 

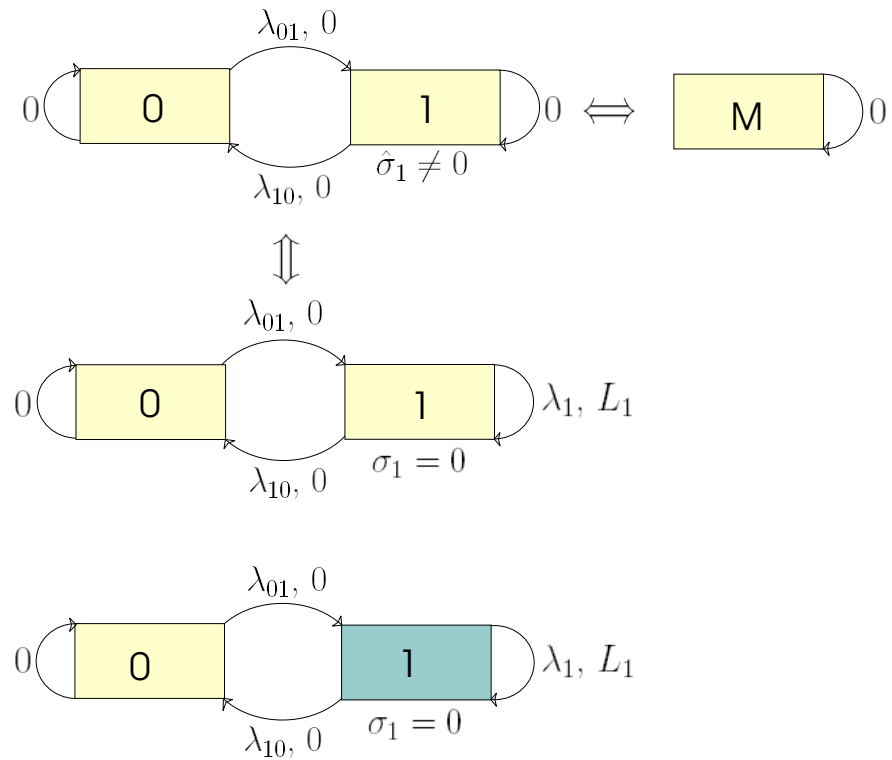

Figure 3: The figure depicts the situation of example two.

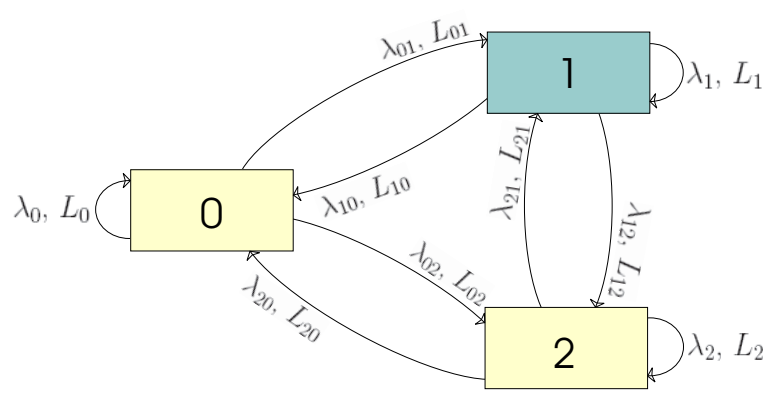

Figure 4: The figure depicts an economy with three regimes where trading is possible in states 0 and 2 and impossible in state 1. 


\begin{tabular}{|c|c|c|c|c|c|c|c|c|c|c|}
\hline$\lambda_{01}$ & $L_{01}$ & $\lambda_{10}$ & $L_{10}$ & $l$ & $T$ & $\pi_{0, i l l i q}^{*}(\%)$ & $\pi_{0, l i q}^{*}(\%)$ & $\Delta x(\%)$ & variable & value \\
\hline \multirow[t]{3}{*}{0.01} & 0 & 0.3 & 0.5 & 0.5 & 10 & 66.34 & 80.00 & 4.72 & $r_{0}$ & 0.03 \\
\hline & & & & & 30 & 66.26 & 80.00 & 13.64 & $r_{1}$ & 0.03 \\
\hline & & & & & 50 & 66.26 & 80.00 & 21.74 & $\alpha_{0}$ & 0.05 \\
\hline 0.01 & 0 & 0.3 & 0.5 & 0 & 50 & 66.26 & 80.00 & 20.68 & $\alpha_{1}$ & $-r_{1}$ \\
\hline \multirow[t]{3}{*}{0.01} & 0 & 0.3 & 0.9 & 0.9 & 10 & 52.29 & 80.00 & 8.27 & $\sigma_{0}$ & 0.25 \\
\hline & & & & & 30 & 52.28 & 80.00 & 22.71 & $\sigma_{1}$ & 0 \\
\hline & & & & & 50 & 52.28 & 80.00 & 34.88 & $\lambda_{0}$ & 0 \\
\hline 0.01 & 0 & 1 & 0.5 & 0.5 & 50 & 67.39 & 80.00 & 21.43 & $\lambda_{1}$ & 0 \\
\hline \multirow[t]{3}{*}{0.02} & 0 & 0.3 & 0.5 & 0.5 & 10 & 55.09 & 80.00 & 8.14 & $L_{0}$ & 0 \\
\hline & & & & & 30 & 54.98 & 80.00 & 22.55 & $L_{1}$ & 0 \\
\hline & & & & & 50 & 54.98 & 80.00 & 34.70 & $t$ & 0 \\
\hline \multirow[t]{3}{*}{0.02} & 0 & 0.3 & 0.9 & 0.9 & 10 & 36.49 & 80.00 & 12.49 & $X(0)$ & 100 \\
\hline & & & & & 30 & 36.47 & 80.00 & 32.67 & & \\
\hline & & & & & 50 & 36.47 & 80.00 & 48.19 & & \\
\hline 0.3 & 0.5 & 0.01 & 0 & 0 & 50 & 0 & 0 & 0 & & \\
\hline 0.01 & 0.5 & 0.3 & 0 & 0 & 50 & 66.26 & 67.89 & 2.16 & & \\
\hline 0.01 & 0.9 & 0.3 & 0 & 0 & 50 & 52.28 & 52.64 & 0.41 & & \\
\hline
\end{tabular}

Table 2: There are two possible states for the economy. The investor maximizes expected indirect logarithmic utility from terminal wealth. The investment horizon is given by $T$. The market consists of a bond with interest rate $r=0.03$ and a stock which is modeled as a diffusion with state dependant volatility $\sigma_{i}$ and excess return $\alpha_{i}$. The intensities for a regime shift from state $i$ to state $j$ are denoted by $\lambda_{i j}$. Upon transition from state $i$ to state $j$, a loss of $L_{i j}$ is involved. If at time $t=T$ the exchange is closed, then the investor can liquidate his shares of the stock only by suffering a loss of rate $l . \pi_{0, i l l i q}^{*}$ and $\pi_{0, l i q}^{*}$ denote the optimal portfolio of the investor when trading is not allowed in state 1 , respectively when trading is allowed in both states. We do not allow for short selling. $\Delta x$ denotes by which amount the initial capital of $X(0)=100$ of an investor who is not allowed to trade in state 1 can be reduced to get the same utility as in the case that trading is allowed in both states. The parameters are chosen to mimic situations such as in Japan after WW II. For instance, the parametrization $\lambda_{01}=0.01$, $\lambda_{10}=0.3, L_{01}=0$, and $L_{10}=0.9$ implies that, on average, once in a century the illiquidity state is reached and, on average, this state is left after 3.33 years triggering a stock price decrease of $90 \%$. In this particular case, for an an investment horizon of $T=30$ years, the change of initial capital amounts to over $22 \%$. 


\begin{tabular}{c|c|c|c|c|c|c|}
$\lambda_{01}$ & $\lambda_{10}$ & $T$ & $\pi_{0, \text { illiq }}^{*}(\%)$ & $\Delta x(\%)$ & variable & value \\
\hline 1 & 0 & 10 & 85.83 & 0.30 & $l$ & 0 \\
& & 30 & 87.88 & 2.19 & $r_{0}$ & 0.03 \\
& & 50 & 89.52 & 4.87 & $r_{1}$ & 0.03 \\
\cline { 1 - 1 } 1 & 0.01 & 30 & 87.69 & 2.02 & $\alpha_{0}$ & 0.05 \\
& & 50 & 89.05 & 4.36 & $\alpha_{1}$ & 0.05 \\
\hline 1 & 0.1 & 30 & 86.28 & 1.14 & $\sigma_{0}$ & 0.25 \\
& & 50 & 86.42 & 2.13 & $\sigma_{1}$ & 0 \\
\hline 0.1 & 0 & 30 & 86.25 & 1.46 & $\lambda_{0}$ & 0 \\
& & 50 & 88.06 & 3.86 & $\lambda_{1}$ & 0.0197 \\
\hline 0.1 & 0.01 & 30 & 85.92 & 1.31 & $L_{0}$ & 0 \\
& & 50 & 87.39 & 3.33 & $L_{1}$ & 0.8 \\
\hline 0.1 & 0.1 & 50 & 83.86 & 1.22 & $L_{01}$ & 0 \\
\hline 0.02 & 0.2 & 50 & 80.76 & 0.19 & $L_{10}$ & 0 \\
\hline 0.01 & 0 & 50 & 82.81 & 1.04 & $t$ & 0 \\
\hline 0.01 & 0.01 & 50 & 82.31 & 0.86 & $X(0)$ & 100
\end{tabular}

Table 3: There are two possible states for the economy. The investor maximizes expected indirect logarithmic utility from terminal wealth. The investment horizon is given by $T$. The market consists of a bond with interest rate $r=0.03$ and a stock which is modeled as a jumpdiffusion with state dependant volatility $\sigma_{i}$, excess return $\alpha_{i}$, relative jump size $L_{i}$ and jump intensity $\lambda_{i}$. The intensities for a regime shift from state $i$ to state $j$ are denoted by $\lambda_{i j}$. If at time $t=T$ the exchange is closed, then the investor can liquidate his shares without any loss. $\pi_{0, \text { illiq }}^{*}$ denotes the optimal portfolio of the investor when trading is not allowed in state 1 . The optimal portfolio of an investor who is allowed to trade in both states is given by $\pi_{0, l i q}^{*}=0.8$. His optimal portfolio when starting in state 1 is given by $\pi_{1, l i q}^{*}=0.855$. We do not allow for short selling. $\Delta x$ denotes by which amount the initial capital of $X(0)=100$ of an investor who is not allowed to trade in state 1 , can be reduced to get the same utility as in the case that trading is allowed in both states. The parameters are chosen such that, in case of trading is allowed in both states, the model is equivalent to a (single-state) Merton model with parameters $\sigma_{M}=0.25, \alpha_{M}=0.05$ and $r_{M}=0.03$. 


\begin{tabular}{|c|c|c|c|c|c|c|c|}
\hline$\lambda_{01}$ & $\lambda_{10}$ & $l$ & $T$ & $\pi_{0, \text { illiq }}^{*}(\%)$ & $\Delta x(\%)$ & variable & value \\
\hline \multirow[t]{3}{*}{1} & 0.01 & 0.5 & 10 & 0 & 18.13 & $r_{0}$ & 0.03 \\
\hline & & & 30 & 57.17 & 37.32 & $r_{1}$ & 0.03 \\
\hline & & & 50 & 74.47 & 41.27 & $\alpha_{0}$ & 0.05 \\
\hline \multirow[t]{3}{*}{1} & 0.1 & 0.5 & 10 & 20.04 & 18.02 & $\alpha_{1}$ & 0.05 \\
\hline & & & 30 & 82.77 & 23.17 & $\sigma_{0}$ & 0.25 \\
\hline & & & 50 & 86.03 & 23.98 & $\sigma_{1}$ & 0 \\
\hline \multirow[t]{3}{*}{0.1} & 0.01 & 0.5 & 10 & 30.44 & 17.32 & $\lambda_{0}$ & 0 \\
\hline & & & 30 & 65.39 & 33.69 & $\lambda_{1}$ & 0.0197 \\
\hline & & & 50 & 75.90 & 38.86 & $L_{0}$ & 0 \\
\hline \multirow[t]{3}{*}{0.1} & 0.1 & 0.5 & 10 & 59.79 & 14.02 & $L_{1}$ & 0.8 \\
\hline & & & 30 & 81.60 & 18.65 & $L_{01}$ & 0 \\
\hline & & & 50 & 83.61 & 19.28 & $L_{10}$ & 0 \\
\hline \multirow[t]{3}{*}{0.01} & 0.01 & 0.5 & 10 & 72.00 & 4.28 & $t$ & 0 \\
\hline & & & 30 & 76.39 & 11.03 & $X(0)$ & 100 \\
\hline & & & 50 & 78.80 & 15.93 & & \\
\hline \multirow[t]{3}{*}{0.01} & 0.01 & 1 & 10 & 50.76 & 9.40 & & \\
\hline & & & 30 & 54.77 & 25.79 & & \\
\hline & & & 50 & 58.52 & 38.74 & & \\
\hline \multirow[t]{3}{*}{0.01} & 0.1 & 0.5 & 10 & 76.76 & 2.93 & & \\
\hline & & & 30 & 80.31 & 4.40 & & \\
\hline & & & 50 & 80.68 & 4.67 & & \\
\hline \multirow[t]{3}{*}{0.01} & 0.1 & 1 & 10 & 64.21 & 6.92 & & \\
\hline & & & 30 & 77.36 & 11.31 & & \\
\hline & & & 50 & 80.21 & 12.08 & & \\
\hline
\end{tabular}

Table 4: The situation is the same as in Table 3. However, if at time $t=T$ the exchange is closed, then the investor can liquidate his shares of the stock only by suffering a loss at the rate $l$. As before, we do not allow for short selling. 